\title{
Trabecular architecture in the StW 352 fossil hominin calcaneus.
}

Angel Zeininger ${ }^{\mathrm{a},{ }^{*}}$, Biren A. Patel ${ }^{\mathrm{b}, \mathrm{c}}$, Bernhard Zipfel $^{\mathrm{c}}$, and Kristian J. Carlson ${ }^{\mathrm{c}, \mathrm{d}}$

${ }^{a}$ Department of Evolutionary Anthropology, Duke University, Box 90383, Durham, NC, 27708, USA.

${ }^{\mathrm{b}}$ Department of Cell and Neurobiology, Keck School of Medicine, University of Southern California, Los Angeles, CA, 90033, USA.

${ }^{c}$ Evolutionary Studies Institute and School of Geosciences, University of Witwatersrand, WITS 2050, Johannesburg, South Africa.

${ }^{\mathrm{d}}$ Department of Anthropology, Indiana University, Bloomington, IN, 47405, USA.

* Corresponding author

E-mail address: angel.zeininger@duke.edu (A. Zeininger)

Keywords: Bipedalism, Foot, Calcaneocuboid joint, Talocalcaneal joint, Trabecular fabric, Midtarsal break

\begin{abstract}
Australopithecus africanus has been interpreted as having a rigid lateral foot. One mechanism contributing to a rigid foot during push-off in humans is a calcaneocuboid joint (CCJ) with limited dorsiflexion and a "close-packed" talocalcaneal joint (TCJ). In contrast, apes
\end{abstract}


likely have a greater CCJ range of motion and lack a close-packed TCJ. Differences in tarsal arthrokinematics may result in different joint loading environments. In Homo sapiens, we tested the hypothesis that dorsal and plantar CCJ and the TCJ show evidence of predictable habitual loading. In Pan troglodytes, Gorilla gorilla, Gorilla beringei, and Papio ursinus, we tested the hypothesis that only the dorsal CCJ shows evidence of predictable loading. Specifically, we predicted similarity in trabecular properties across the dorsal and plantar CCJ in $H$. sapiens, but dissimilarity in non-humans. Additionally, we investigated trabecular properties of an $A$. africanus calcaneus (StW 352) to evaluate joint loading patterns in this hominin and ultimately address the evolution of these properties in $H$. sapiens. Contrary to predictions, the $H$. sapiens dorsal CCJ has a significantly higher elongation index, bone volume fraction, trabecular thickness, and trabecular number than the plantar CCJ, while trabecular properties in nonhumans do not always differ as predicted between regions. Homo sapiens exhibits trabecular morphology indicative of less variable TCJ loading than other groups, having the most anisotropic and rod-like struts oriented in line with predicted principal loads. Multivariate analysis shows that the StW 352 dorsal CCJ matches P. ursinus best, while the plantar CCJ matches $G$. beringei best and the TCJ matches that of $G$. gorilla best. Overall patterns suggest that the StW 352 calcaneus experienced more variable loading than H. sapiens, but less variable loading than P. troglodytes, G. gorilla, G. beringei, and P. ursinus, consistent with a large range of foot movements, probably reflecting locomotor kinematics that are unlike those of living humans or apes. 


\section{Introduction}

The partial right calcaneus, StW 352, from Member 4 of Sterkfontein, South Africa, is missing only the posterior portion of its calcaneal tuber (Fig. 1). Despite being incomplete, it still represents one of the better-preserved calcaneal specimens in the hominin fossil record. Although the presence of at least two australopith species in Member 4 has been suggested (Clarke, 2013), this specimen is most commonly attributed to Australopithecus africanus (Deloison, 2003; Prang, 2015). Deloison (2003) originally described StW 352 as having an enlarged peroneal process and a human-like robust tuber, longitudinal axis, and cuboid facet inclination angle. Based in part on the latter constellation of characters, she suggested that $A$. africanus (as represented by StW 352) likely had an elevated calcaneocuboid joint resulting from a human-like plantar arch, and thus inferred a human-like foot function (Deloison, 2003, 2004). In a more recent study, however, Prang (2015) concluded that the remaining part of the calcaneal tuber of StW 352 is relatively gracile and more chimpanzee-like than previously thought. Moreover, Prang (2015) observed that the cross-sectional area of the remaining part of the calcaneal tuber of StW 352 was more similar to that of Australopithecus sediba from Malapa (Zipfel et al., 2011) and less robust than Australopithecus afarensis calcanei from Hadar (Latimer and Lovejoy, 1989). He suggested that this demonstrated evidence that later australopiths from South Africa (A. africanus and A. sediba) were less well-adapted to terrestrial bipedalism than earlier australopiths from East Africa (A. afarensis). Thus, resolving the conflicting observations of the extent of human-like qualities in StW 352 may have significant implications for interpreting the evolution of bipedalism within the hominin clade. To further evaluate hominin calcaneal morphology, and hopefully shed light on the functional basis for 
these opposing views, the aim of this study is to investigate an additional aspect of the StW 352 calcaneus, its trabecular structure, and interpret it within a comparative context using a sample of humans (Homo sapiens), African apes (Pan troglodytes, Gorilla gorilla, and Gorilla beringei), and baboons (Papio ursinus).

Calcaneal morphology beyond absolute and relative measures of tuber robusticity can be informative when reconstructing hominin foot function. For example, analyzing functional morphology of the StW 352 distal cuboid articular facet (i.e., the articulation with the cuboid at the calcaneocuboid joint $[\mathrm{CCJ}])$ may indicate whether this individual had a relatively more stable (i.e., rigid) or a more mobile midfoot, the latter condition being considered more characteristic of apes and monkeys, and the former condition being considered more characteristic of modern humans (Elftman and Manter, 1935a; Morton, 1935; Bojsen-Møller, 1979; Susman, 1983). In contrast to humans, both apes and monkeys dorsiflex their midfoot following flat foot and during the push-off phase of bipedal or quadrupedal locomotion. Although the CCJ has traditionally been viewed as the site of dorsiflexion in the midfoot (Elftman and Manter, 1935a; Susman, 1983), more recent work (D’Aout et al., 2002; Vereecke et al., 2003; DeSilva, 2010; Bates et al., 2013; Thompson et al., 2014; DeSilva et al., 2015) suggests that initial dorsiflexion occurs at the CCJ followed by additional dorsiflexion at the talonavicular joint and more substantial dorsiflexion at the tarsometatarsal joint between the cuboid and fourth and fifth metatarsals.

Non-human primates can lift the heel independently of the rest of the foot, known as the 'midtarsal break', because of movement capability in several joints (Elftman and Manter, 1935b). Dorsiflexion of the foot involves a number of joints, one of which, the CCJ, makes an important contribution to this midtarsal break. Kinematic and cineradiographic data (e.g., DeSilva et al., 2010; Thompson et al., 2013) demonstrate that greater amounts of midtarsal break 
occur in the CCJ of P. troglodytes, G. gorilla, and Papio compared to H. sapiens. Based on lateral view video footage of captive apes performing slow quadrupedal walking gaits on flat, concrete surfaces, DeSilva (2010) measured a mean CCJ dorsiflexion angle of $16.1^{\circ}$ in $P$. troglodytes and $14.1^{\circ}$ degrees in G. gorilla. Thompson and colleagues (2014) correspondingly reported mean dorsiflexion of $14^{\circ}$ at the $\mathrm{CCJ}$ using cineradiography during passive foot movements in anesthetized $P$. troglodytes. By comparison, approximately $2.3^{\circ}$ of dorsiflexion in the H. sapiens CCJ has been reported (Ouzounian and Shereff, 1989). Subchondral bone radiodensity data are also consistent with results from kinematic and cineradiography studies: $P$. troglodytes and $P$. ursinus exhibit relatively more expansive areas of high radiodensity (a proxy for higher compressive strength) in the dorsal region of the cuboid facet compared to its plantar region, in contrast to $H$. sapiens, in which there are no significant differences in areas of relatively high radiodensity between the dorsal and plantar regions (Nowak et al. 2010).

While subchondral bone apparent density patterns have proven useful in documenting the loading history of joints in extant animals (Carlson and Patel, 2006; Patel and Carlson, 2007, 2008; Polk et al., 2008; Nowak et al., 2010; Su, 2011; Carlson et al., 2013), there are obstacles in applying this approach to fossils. Namely, local variation in diagenetic processes can differentially affect mineralization of a joint region. Thus, there is no guarantee after mineral exchange during fossilization that density gradients in a fossil reflect those that were present in vivo (Patel and Carlson, 2007; Polk et al., 2010). Evaluating the trabecular bone deep to the subchondral region of a joint surface of a fossil offers a useful alternative for linking calcaneal joint anatomy with joint movement and loading history (e.g., Su, 2011; Zeininger et al., 2011). Trabecular bone, a network of tiny struts (i.e., individual trabeculae) positioned deep to the cortical shell, is found throughout the skeleton, including within a significant proportion of 
tarsals (Currey, 1984). Bone modeling over the lifetime of an individual has been shown, in some cases, to align trabeculae with the direction of principal loads (Meyer, 1867; Wolff, 1892; Odgaard et al., 1997; Pontzer et al., 2006; Barak et al., 2011). Experimental and modeling data suggest that there are correlations between external loading, trabecular fabric properties, and trabecular elastic properties (e.g., Young's modulus and shear modulus; Hodgskinson and Currey, 1990; Turner et al., 1990; Goldstein et al., 1991; Odgaard et al., 1997; Kabel et al., 1999; Ulrich et al., 1999).

Caution is warranted since not all characterizations of trabecular architecture deep to joint surfaces show the predicted functional signals (e.g., Ryan and Walker, 2010). However, these signals appear to be relatively more robust when considering multiple trabecular properties in a multivariate approach (Ryan and Shaw, 2012). Also, previous studies of trabecular bone morphology in the foot have presented conflicting results with respect to predicted muscle and joint loading patterns, with some finding significant bipedal locomotor correlates (calcaneus: Maga et al., 2006; talus: Su et al., 2013, Su and Carlson, in review; metatarsal heads: Griffin et al., 2010), but other analyses (calcaneus: Kuo et al, 2013; talus: DeSilva and Devlin, 2012) failing to differentiate between groups.

During walking gaits, humans have a relatively rigid lateral midfoot with minimal interbone movements during the push-off phase of stance. During load acceptance in the early part of stance phase, subtalar and transverse tarsal joints (talonavicular and CCJ) are in a loose-packed position (MacConnail and Basmajian, 1969; Sarrafian, 1993; Sammarco, 2004). At this time the posterior articular facets of the talus and calcaneus are misaligned. The transverse tarsal joints, on the other hand, are aligned (i.e., their axes are parallel in the coronal plane), allowing for greater dorsiflexion/plantarflexion movement in the sagittal plane. As the ankle dorsiflexes and 
body weight passes over the foot, the subtalar joint inverts, bringing the talocalcaneal joint (TCJ) to a "close-packed" position (Sammarco, 2004). However, the transverse tarsal joints simultaneously become misaligned (i.e., their axes are no longer parallel), preventing dorsiflexion/plantarflexion movement in the sagittal plane. Concurrent exorotation of the calcaneus causes the calcaneus to swing laterally, close-packing the CCJ (Sarrafian, 1993). Thus, in humans, both the TCJ and CCJ are close-packed from midstance until the end of stance phase, transforming the foot into a rigid lever in preparation for push-off (Sammarco, 2004). Finite element analyses (FEA) of the calcaneus predict that, at heel strike and in preparation for pushoff, summed force across the TCJ is elevated (3.1 times body weight at impact and 5.4 times body weight at $70 \%$ of stance phase; Giddings et al., 2000). Maximum force at both the TCJ and CCJ (4.2 times body weight) is predicted at $70 \%$ of stance phase in human walking, when both joints are close-packed and the foot propels the center of mass forward (Giddings et al., 2000). Close-packing of the TCJ is not thought to occur during push-off in non-human primates and is, therefore, considered unique to humans (Elftman and Manter, 1935a; Close et al., 1967; Lewis, 1980; Langdon et al., 1991).

Close-packing of joints is thought to be associated with the need to accommodate high compressive forces. Since joint surfaces are maximally congruent during close-packing, compressive forces are theoretically distributed over a large surface area, thereby minimizing the peak compressive force per unit area at any particular location. Thus, the loading environment of trabecular bone associated with a close-packed joint should be one of habitual load orientation (i.e., limited movement at the joint resulting in more uniform strut orientations) and relatively lower compressive forces at any given location (i.e., experienced by any individual strut) compared to a joint that is not close-packed. Trabecular strut shape, measured as the elongation 
index, provides some support for this idea in that it has been shown to differ between loading environments characterized by relatively high force (i.e., low elongation index, disc-like struts) versus relatively low force (i.e., high elongation index, rod-like struts; Ding et al., 2002).

In the current study, we test the hypothesis that habitual joint forces (i.e., loading regimes) acting on the distal calcaneus during push-off are preserved in trabecular architecture deep to the cuboid facet in H. sapiens, P. troglodytes, G. gorilla, and P. ursinus. We limited our ape sample to African apes as they are the closest living relatives to humans and are more terrestrial than orangutans or hylobatids, which are generally more specialized for suspensory locomotor behaviors (i.e., their foot is used differently to interact with more compliant substrates and superstrates). Additionally, we included Papio in our sample since they are amongst the most terrestrial non-hominoid primates, are relatively large in body size, and because the morphology and function of the CCJ has been documented in this group (DeSilva, 2010; Nowak et al., 2010). We then use these modern comparative data to interpret trabecular structure and calcaneal loading in StW 352. We predict that the dorsal aspect of the cuboid facet in $P$. troglodytes, G. gorilla, G. beringei, and P. ursinus will show evidence of higher compressive joint loads (e.g., greater bone volume fraction [BV/TV] and more disc-like struts in underlying trabecular bone) relative to the plantar aspect of the facet. In contrast, we predict that BV/TV, elongation index, and strut orientation (e.g., degree of anisotropy) across dorsal and plantar regions deep to the $H$. sapiens cuboid facet will not differ appreciably. Finally, we predict that StW 352, unlike H. sapiens, will exhibit some degree of dorsal versus plantar differentiation in $\mathrm{CCJ}$ trabecular architecture if this individual was not as well-adapted to terrestrial bipedalism as H. sapiens, as has been recently suggested (Prang, 2015). 
Because StW 352 preserves its posterior talar articular facet, its functional morphology also can be evaluated. However, since we are unaware of specific in vivo studies of TCJ arthrokinematics in nonhuman primates, or studies of subchondral bone apparent density patterns in the TCJ, we avoid specific regional predictions of trabecular bone structure (e.g., dorsal versus plantar BV/TV), instead favoring those reflecting theoretical loading conditions during overall TCJ close-packing. Because the TCJ is thought to achieve a close-packed position only in $H$. sapiens, we predict that compared to P.troglodytes, G. gorilla, G. beringei, and P. ursinus, humans will have a higher degree of anisotropy (i.e., more highly-oriented trabecular struts due to less variable movement in this joint), higher elongation index (i.e., more rod-like trabeculae), and that the trabeculae will be more oriented along the anteroposterior (A-P) plane (i.e., in line with the predicted joint reaction forces; Fig. 2). Once again, we predict that StW 352 will be unlike humans or apes, but rather will exhibit a pattern that is intermediate between the two.

\section{Materials and methods}

Human calcanei were obtained from the Norris Farms collection (\#36 cemetery), housed at Pennsylvania State University $(n=5)$, and the Raymond A. Dart Collection of Human Skeletons, housed at the University of the Witwatersrand, Johannesburg, South Africa $(n=10$ Zulu individuals). Materials from the Norris Farms Oneota culture are on loan from the Illinois State Museum and date to 1300 AD (Milner and Smith, 1990). Wild caught, non-pathological adult (as determined by the dental age defined in Shea [1981]) chimpanzee (P. troglodytes; $n=$ 18) and gorilla ( $n=10 \mathrm{G}$. gorilla and $n=6 \mathrm{G}$. beringei beringei) calcanei were sampled from the American Museum of Natural History (New York, NY) and the National Museum of Natural 
History (Washington, DC). Gorilla species were separated in all analyses since recent studies of foot bones have found significant ecomorphological differences in mountain and lowland species (Tocheri et al., 2011; Dunn et al., 2014). Baboon calcanei (P. ursinus; $n=11)$ were obtained from adult individuals (determined by long bone epiphyseal fusion) in the Raymond A. Dart Collection of comparative skeletal material, housed at the University of Witwatersrand. For all species, males and females were pooled and samples also included a small number of individuals of unknown sex.

Homo sapiens calcanei from the Norris Farms collection were CT scanned on the HD600 high-resolution industrial X-ray CT scanner at the Center for Quantitative Imaging, Pennsylvania State University. Additional H. sapiens and all P. ursinus calcanei, as well as StW 352, were scanned at the Microfocus X-ray CT Facility of the Palaeosciences Centre at the University of the Witwatersrand. The StW 352 calcaneus was scanned at $90 \mathrm{kV}$ and $135 \mu \mathrm{A}$, using 5600 projections with two frame averaging. A portion of the P. troglodytes. G. gorilla, and G. beringei sample was scanned at the High Resolution X-ray CT Facility at the University of Texas-Austin. The remaining African ape sample was scanned at the University of Southern California's Molecular Imaging Center using a SCANCO Medical $\mu$ CT 50 specimen scanner, a GE Pheonix Nanotom M scanner, or a Nikon XT H 225 scanner. All extant bones were CT scanned at the highest possible spatial resolution available for their size (26 to $56 \mu \mathrm{m})$. Image data were obtained from StW 352 using $29.6 \mu \mathrm{m}$ voxel resolution. A customized segmentation technique was performed in Avizo Fire 8.1 software to remove internal matrix from the StW 352 specimen. Material contrast between fossilized bone comprising struts and the matrix filling intervening trabecular spaces allowed adequate separation of the two materials in StW 352 (Fig. $3)$. 
All image stacks were converted from 16-bit TIFF (or DICOM) images to 8-bit TIFF images in ImageJ software (Rasband, 1997-2014), thereby reducing gray levels from 65,536 to 256 without affecting spatial resolution. Using Quant3D software (Ketcham and Ryan, 2004), trabeculae were sampled within three spherical volumes of interest (VOI) in each calcaneus (Fig. 3): one VOI was positioned deep to the posterior talar articular facet at the TCJ and two VOIs were positioned deep to the cuboid articular facet at the CCJ (i.e., dorsal and plantar CCJ VOIs). Spherical VOIs were chosen over cubic VOIs in order to avoid corner edge effects (Ketcham and Ryan, 2004). Each VOI was scaled to joint size for each specimen. Joint size was measured on three-dimensionally reconstructed image stacks in Avizo 9.0 software (FEI, Hillsboro, OR, USA). For the TCJ, VOI diameter was calculated as one-half the height of the talar articular facet. For the CCJ, each VOI was calculated as one-third the height of the cuboid facet. Small VOIs may not satisfy the continuum assumption (Hoffler et al., 2000) and, therefore, all VOIs were visually inspected to ensure that they contained at least five trabecular struts (Harrigan et al., 1984). Diameters of VOIs ranged in size from $3.28 \mathrm{~mm}$ to $16.15 \mathrm{~mm}$ (Table 1).

An effort was made to place VOIs in biomechanically homologous locations (Fajardo and Mueller, 2001; Maga et al., 2006; Lazenby et al., 2011; Kivell et al., 2011; Su et al., 2013; Su and Carlson, in review) despite challenges created by shape differences across taxa. Although defining the transition between trabecular struts and overlying cortical bone or densely-packed subchondral bone can be difficult, each VOI was conservatively placed as close to the perimeter of the bone as possible while still ensuring that the three-dimensional VOI contained only trabecular struts. Due to the thick subchondral bone at the TCJ in P. ursinus (Fig. 3), we could not confidently identify an appropriately-sized VOI containing only trabecular struts deep to the facet. As such, we excluded P. ursinus from the TCJ analyses. 
Since image data from calcanei were not always acquired with the bones in anatomical (or at least a single consistent) position, anatomical axes were manually re-defined for each bone prior to analysis. A separate threshold was applied to each VOI using an iterative segmentation algorithm (Ridler and Calvard, 1978; Trussel, 1979). The star volume distribution (SVD) method was used to calculate trabecular fabric properties of each VOI. The SVD method measures trabecular fabric anisotropy by calculating the distance between trabecular struts in various dimensions throughout a stack of 8-bit TIFF images. Briefly, a set of points are randomly selected from within a VOI. Cones are drawn from each point and stopped when they encounter trabecular struts, resulting in a numerical quantification of anisotropy in the form of a $3 \times 3$ orientation matrix. Primary, secondary, and tertiary trabecular orientations and magnitudes are represented by the eigenvectors (direction) and eigenvalues (magnitude) of the matrix (Odgaard, 1997; Ketcham and Ryan, 2004). These eigenvectors and eigenvalues are used to determine trabecular fabric properties. Primary and tertiary eigenvalues are used to calculate the degree of anisotropy (DA) within a VOI (i.e., DA $=1$-[tertiary/primary eigenvalue]) on a scale from $0-1$ where a DA equal to 0 represents a perfectly isotropic structure and a DA of 1 represents a perfectly anisotropic structure. Elongation index (E) is defined as 1-(secondary/primary eigenvalue). When DA is near 1, a high E value represents rod-shaped trabecular struts, while a low E value represents more disc-shaped struts. Bone volume fraction (BV/TV) is calculated as the ratio of bone voxels (BV) to total voxels (TV) in a given VOI, where a high BV/TV ratio indicates densely-packed trabecular struts. Trabecular thickness (Tb.Th) is measured as the mean minimum measured length through a random point within bone (Ketcham and Ryan, 2004). Trabecular number (Tb.N) was estimated, using the mean intercept length (MIL) method in 
Quant3D, as the number of intersections between a grid of lines and the bone, and then normalized by total grid-line length.

Papio ursinus calcanei (originally scanned at $26 \mu \mathrm{m}$ ) were used to assess resolution dependency in this sample because it was most important to ensure that resolution was sufficiently high in the smallest bones. A VOI was extracted from each calcaneus and then down-sampled to one higher (20) and four lower (30,40, 50, and $60 \mu \mathrm{m})$ spatial resolutions using the Resample module with a Lanczos filter in Avizo 7.1, ultimately encompassing the full range of resolutions used in this study (Ryan and Shaw, 2012). A least squares linear regression was run for Tb.Th, which can be resolution-dependent (Ryan and Shaw, 2012). Trabecular thickness was not significantly correlated with voxel size $(p>0.05)$ in this sample of calcanei and, thus, raw Tb.Th values were used in all analyses.

To visually assess differences across all taxa, boxplots and stereoplots were generated. Boxplots visually compare standard properties. Stereoplots with 95\% confidence ellipses visually compare trabecular orientation. To test for statistically significant group differences in these properties, Kruskal-Wallis tests were run for each trabecular fabric property, followed by pairwise Mann-Whitney U tests. To further test for statistically significant differences between dorsal and plantar VOIs within a taxon, Wilcoxon tests were used. All statistical analyses were conducted in PAST (Hammer et al., 2001), with statistical significance determined by an alpha $\leq$ 0.05 .

To further investigate whether trabecular bone properties deep to each joint surface (TCJ, dorsal CCJ, plantar CCJ, and combined dorsal and plantar CCJs) can discriminate between taxonomic groups, four variables were subjected to discriminant function analyses: Tb.Th, Tb.N, DA, and E. Each extant group (H. sapiens, P. troglodytes, G. gorilla, G. beringei, and $P$. 
ursinus) was assigned to its own group a priori, while Stw 352 was not assigned a priori to any group, nor to its own group (i.e., A. africanus). The classification results (i.e., percent correct classifications or posterior probabilities) were evaluated to compare actual group membership to predicted group membership (e.g., Patel, 2005; Ryan and Shaw, 2012; Scherf et al., 2015).

Because the original discriminant function analyses may exaggerate estimates of classification, cross-validation tests were performed to assess the performance of the discriminant functions in measuring percent correct classifications. During cross-validation, each case or specimen in the analysis is classified by the discriminant functions derived from all cases excluding itself (i.e., the leave-one-out test was employed). The percent correct classification results of the original discriminant function analyses were then compared to the cross-validation results. If differences were great, this would suggest that trabecular properties used in the analyses are weak discriminators for taxonomic identification. Separation of groups was also evaluated by the analysis of structure coefficients (i.e., the pooled within-group correlation between the predictor variable and the discriminant score). Discriminant function analyses were performed with SPSS v. 16.0 software.

\section{Results}

\section{Calcaneocuboid joint}

Dorsal VOI: Extant sample $H$. sapiens exhibited the lowest mean BV/TV in this region (Fig. 4, Table 2), significantly lower than those of P. troglodytes $(p<0.001)$ and G. gorilla $(p<0.05)$. Mean trabecular thickness was highest in G. gorilla and lowest in P. ursinus (Fig. 4, Table 2), 
differing significantly across extant taxa $(\mathrm{H}=15.55, p=0.003)$. Gorilla gorilla exhibited significantly higher Tb.Th than $H$. sapiens $(p=0.005)$, . troglodytes $(p=0.033)$, and $P$. ursinus ( $p<0.001)$, while G. beringei exhibited significantly higher Tb.Th than P. ursinus $(p=0.007$; Table 3). Mean Tb.N was highest in P. troglodytes and lowest in G. gorilla (Fig. 4, Table 2), differing significantly across extant taxa $(\mathrm{H}=9.55, p=0.049)$.

Mean DA in the dorsal CCJ VOI was highest in H. sapiens and lowest in Pan (Table 2), differing significantly across extant taxa $(\mathrm{H}=37.24, \mathrm{p}<0.001$; Fig. 4, Table 3$)$. H. sapiens had significantly higher DA than all other taxa $(p<0.001)$. In addition, DA in $P$. troglodytes was significantly lower than in G. gorilla $(p=0.022)$ and P. ursinus $(p=0.005)$. Homo sapiens had not only the highest DA values, but also the highest E values, significantly higher than all other taxa $(p<0.001$; Table 3$)$ and, thus, the most anisotropic and rod-like trabecular struts in the dorsal region of the CCJ. Primary trabecular orientation was generally in the A-P direction in all extant taxa, with the exception of a single $H$. sapiens and two P. troglodytes outliers (Fig. 5). Gorilla beringei displayed the most variability of extant groups, and emphasized mediolateral variability in primary orientation, while $P$. troglodytes and $H$. sapiens to a lesser extent varied along the dorsoplantar axis more than other taxa.

The multivariate discriminant function analysis produced four discriminant functions with Functions 1 and 2 accounting for $57.8 \%$ and $41.1 \%$ of trabecular variance, respectively (Table 4). Function 1 was most highly correlated with E and DA, while Function 2 was most highly correlated with Tb.N and Tb.Th (Table 5). Of the 60 individuals in this study, only 55\% of the cross-validated grouped cases were correctly classified (Table 6). Approximately $93 \%$ of H. sapiens were classified correctly, while only $67 \%$ of P. troglodytes, $70 \%$ of G. gorilla, and 
$36 \%$ of $P$. ursinus were classified correctly (Table 6). Individuals within the small sample of $G$. beringei were most often misclassified as G. gorilla (50\%; Table 6).

Dorsal VOI: StW 352 Bone volume fraction of StW 352 was closest to the H. sapiens mean, falling within the lower ends of $H$. sapiens, G. gorilla, G. beringei, and P. ursinus ranges, but below the $P$. troglodytes range (Fig. 4, Table 2). In Tb.Th, StW 352 exceeded all extant group means except G. gorilla and G. beringei (Fig. 4, Table 2). Trabecular thickness of StW 352 was within the lower end of the G. gorilla and G. beringei range, within the upper end of the $H$. sapiens and $P$. troglodytes ranges, and above the P. ursinus range (Fig. 4, Table 2). In Tb.N, StW 352 was considerably lower than each extant group mean, but was still within the lower end of the $P$. troglodytes and G. gorilla ranges (Fig. 4, Table 2). Most similar to the pattern observed in G. gorilla, StW 352 exhibited relatively few but thick trabecular struts in the dorsal region of the CCJ. In DA, StW 352 was most similar to G. beringei. StW 352 fell at the upper end of the $P$. troglodytes DA range, at the lower end of the G. gorilla, G. beringei, and P. ursinus DA ranges, and well below the H. sapiens DA range (Fig. 4, Table 2). Overall trabecular shape in StW 352 was relatively rod-like (relatively high E), being most similar to P. ursinus (Fig. 4, Table 2). StW 352 fell within the upper end of $P$. troglodytes, G. gorilla, and G. beringei E ranges, but below the $H$. sapiens $\mathrm{E}$ range (Table 2). Homo sapiens and $P$. ursinus patterns, which pair high DA and E, provide the best extant comparisons for the relatively anistropic and rod-like struts in StW 352. Primary trabecular orientation of StW 352 was also A-P, similar to all extant taxa, but fell specifically only within the G. beringei $95 \%$ confidence interval (Fig. 5). For the dorsal CCJ VOI, the discriminant function analysis predicted $\mathrm{StW} 352$ to be most similar to $P$. ursinus (0.469; Table 6). 
Plantar VOI: Extant sample Homo sapiens exhibited the lowest mean BV/TV of all groups, while G. gorilla exhibited the highest (Fig. 4, Table 2). Significant differences across extant taxa were observed $(\mathrm{H}=32.69, p<0.001)$, particularly in the case of $H$. sapiens that had a significantly lower mean than P. troglodytes $(p<0.001)$, G. gorilla $(p<0.001)$, G. beringei $(p=$ 0.004), and P. ursinus ( $p<0.001$; Table 3$)$. In addition, the $P$. ursinus mean was significantly lower than that of P. troglodytes $(p=0.016)$ and G. gorilla $(p=0.047$; Table 3). Gorilla gorilla exhibited the highest Tb.Th, while H. sapiens and P. ursinus exhibited the lowest (Fig. 4, Table 2). Trabecular thickness differed significantly across extant taxa $(H=19.27, p<0.001)$, where G. gorilla had significantly higher Tb.Th than H. sapiens $(p=0.001), P$. troglodytes $(p=0.009)$, and P. ursinus ( $p<0.001$; Table 3$)$. This is the same pattern as observed in the dorsal CCJ VOI. Mean Tb.N was highest in P. troglodytes and lowest in H. sapiens (Fig. 4, Table 2), differing significantly across extant taxa $(\mathrm{H}=28.35, p<0.001$; Table 3$)$. Homo sapiens mean Tb.N was significantly lower than in P. troglodytes $(p<0.001)$, G. gorilla $(p=0.002)$, G. beringei $(p=$ 0.011), and P. ursinus ( $p<0.001)$, while P. ursinus mean Tb.N was significantly higher than in G. beringei $(p=0.012$; Table 3). Low BV/TV values in the plantar region of the $H$. sapiens CCJ appear to reflect relatively few and moderately thin trabecular struts, while G. gorilla, on the opposite end of the spectrum, had relatively few, but thick trabecular struts deep to the plantar region of the CCJ.

In the plantar CCJ VOI, DA was highest in H. sapiens and lowest in G. beringei (Fig. 4, Table 2), differing significantly across extant taxa $(\mathrm{H}=23.85, p<0.001$; Table 3$)$. Homo sapiens drove the difference, being significantly higher than all other extant taxa: $P$. troglodytes $(p<0.001)$, G. gorilla $(p<0.001)$, . beringei $(p=0.002)$, and P. ursinus $(p=0.001$; Table 3$)$. 
Primary trabecular orientation was in the A-P plane in all extant taxa, except for a few outliers that exhibited a relatively dorsally-oriented primary trabecular orientation (Fig. 5).

Discriminant function analysis produced four discriminant functions with Function 1 accounting for $75.9 \%$ of trabecular variance, and Functions 2 and 3 accounting for $15.3 \%$ and $7.8 \%$, respectively (Table 4). Function 1 was most highly correlated with Tb.N and DA (Table 5). Function 2 was most highly correlated with Tb.Th and Tb.N (Table 5). Functions 3 and 4 were most highly correlated with E and DA (Table 5). Of the 60 individuals in this study, 55\% of the cross-validated grouped cases were correctly classified (Table 6). While only $64 \%$ of $P$. ursinus, $61 \%$ of $P$. troglodytes, and $60 \%$ of G. gorilla were classified correctly, $80 \%$ of the $H$. sapiens sample was correctly classified (Table 6). Once again, individuals within the small sample of G. beringei were most often misclassified as G. gorilla (50\%; Table 6).

Plantar VOI: StW 352 BV/TV exceeded all extant group means, but was closest to the $G$. gorilla mean (Fig. 4, Table 2). For StW 352, BV/TV fell at the upper end of the P. troglodytes, G. gorilla, and G. beringei ranges, and above the upper end of the H. sapiens and P. ursinus ranges (Fig. 4, Table 2). Trabecular thickness in StW 352 also exceeded all extant group means, but again was closest to the G. gorilla mean (Fig. 4, Table 2). For StW 352, Tb.Th fell within the upper end of the H. sapiens, P. troglodytes, G. gorilla, and G. beringei ranges, and above the upper end of the P. ursinus range (Fig. 4, Table 2). In StW 352, Tb.N was lower than all extant group means except $H$. sapiens and $G$. beringei (Fig. 4, Table 2), but was still within the range for all extant groups (Fig. 4, Table 2). Similar to the dorsal region of the CCJ, and again most like the pattern in G. gorilla, StW 352 had relatively fewer, thicker trabecular struts deep to the plantar region of the CCJ. In StW 352, DA was uniquely low compared to extant group means, 
falling below the ranges of all extant groups. Additionally, elongation index in StW 352 fell below extant group means (Fig. 4, Table 2), suggesting that trabecular struts in the plantar region of the StW 352 CCJ were relatively more isotropic and disc-like than in extant groups. Gorilla beringei exhibited the most similar pattern to $\mathrm{StW} 352$ amongst the extant groups in exhibiting the lowest mean DA and E (Table 2). Primary trabecular orientation of StW 352 was also relatively A-P, but fell outside the $95 \%$ confidence intervals of any extant group (Fig. 5). In the DFA for the plantar CCJ VOI, StW 352 had a predicted probability of being most similar to $G$. beringei (0.494) and G. gorilla (0.444; Table 6).

Combined VOIs In directly comparing dorsal and plantar CCJ VOIs, E $(p<0.001), \mathrm{BV} / \mathrm{TV}(p<$ $0.001)$, Tb.Th $(p=0.008)$, and Tb.N ( $p<0.001)$ were all significantly higher in $H$. sapiens in the dorsal CCJ VOI (Table 7). In P. troglodytes, Tb.Th $(p=0.049)$ was significantly higher in the dorsal than the plantar CCJ VOI, while Tb.N ( $p=0.025$ ) was significantly lower (Table 7). In $G$. gorilla, only DA was significantly higher $(p=0.041)$ in the dorsal than the plantar CCJ VOI (Table 7). In P. ursinus, DA $(p=0.037)$ and $\mathrm{E}(p=0.041)$ were significantly higher in the dorsal than the plantar CCJ VOI (Table 7). In contrast to these groups, there were no significant differences between BV/TV, Tb.Th, Tb.N, DA, or E in the dorsal and plantar CCJ VOIs of $G$. beringei (Table 7). In StW 352, BV/TV, Tb.Th, and Tb.N were lower in the dorsal VOI than the plantar VOI, while DA and E were higher in the dorsal VOI. The overall pattern of dorsal versus plantar VOI differences in StW 352 did not match a taxon-specific pattern amongst the extant groups.

When combining results from dorsal and plantar CCJ VOIs, the discriminant function analysis produced four discriminant functions with Function 1 accounting for $64.1 \%$ of 
trabecular variance, and Functions 2 and 3 accounting for $25.7 \%$ and $8.8 \%$, respectively (Table 4). Function 1 was most highly correlated with $\mathrm{E}$ and $\mathrm{DA}$ in the dorsal CCJ, and Tb.N and DA in the plantar CCJ. Function 2 was most highly correlated with $\mathrm{Tb} . \mathrm{N}$ and $\mathrm{Tb}$.Th in both the dorsal and plantar CCJ. Function 3 was most highly correlated with E and DA in both the dorsal and plantar CCJ (Table 5). Of the 60 individuals in this study, 56.7\% of the cross-validated grouped cases were correctly classified, which was slightly higher than for either the dorsal or plantar CCJ VOI alone (Table 6). As in the separate dorsal and plantar VOI analyses, H. sapiens always had the best classification rates (93\%). Compared to separate dorsal and plantar VOI analyses, $P$. ursinus (82\%), P. troglodytes (72\%), and G. gorilla (70\%) were more often classified correctly in the combined CCJ analysis. Gorilla beringei was again often misclassified as G. gorilla (67\%; Table 6). For the combined CCJ analysis, StW 352 had a predicted probability of being most similar to P. ursinus (0.552; Table 6).

Talocalcaneal joint

TCJ VOI: Extant sample Homo sapiens exhibited the lowest mean BV/TV, while P. troglodytes exhibited the highest (Fig. 4, Table 8). Group differences were significant $(\mathrm{H}=18.34, p<$ $0.001)$, with $H$. sapiens being significantly lower than all other taxa: $P$. troglodytes $(p<0.001)$, G. gorilla $(p=0.015)$, and G. beringei $(p=0.035$; Table 3$)$. Despite these differences in $\mathrm{BV} / \mathrm{TV}$, trabecular $\mathrm{Tb} . \mathrm{Th}(\mathrm{H}=6.03, p=0.108)$ did not differ significantly amongst extant groups (Table 3). Tb.N differed significantly across extant groups $(\mathrm{H}=9.23, p=0.026)$, being significantly lower in G. gorilla than P. troglodytes ( $p=0.010$; Tables 3 and 8). 
Homo sapiens exhibited the highest mean DA, while G. beringei exhibited the lowest mean DA (Fig. 4, Table 8). Degree of anisotropy differed significantly across extant groups (H $=25.67, p<0.001$; Table 3 ), which appears to be driven by $H$. sapiens differing significantly from P. troglodytes ( $p<0.001)$, G. gorilla $(p=0.005)$, and G. beringei $(p=0.002$; Table 3$)$. Gorilla gorilla had significantly higher DA than P. troglodytes $(p=0.016)$ and G. beringei $(p=$ 0.039). H. sapiens exhibited the highest mean elongation index, while $P$. troglodytes exhibited the lowest (Table 8). Elongation index differed significantly across taxa $(\mathrm{H}=18.64, p<0.001)$, with $H$. sapiens having significantly higher E than $P$. troglodytes $(p<0.001)$ and $G$. beringei $(p$ $=0.006)$, and $G$. gorilla having significantly higher E than P. troglodytes $(p=0.006)$ and $G$. beringei $(p=0.026)$. Primary trabecular orientation in all groups was A-P with the exception of single H. sapiens and P. troglodytes outliers in the dorsoplantar plane (Fig. 5).

Discriminant function analysis produced three discriminant functions (Table 4). Function 1 accounted for $79.8 \%$ of trabecular variance, Function 2 accounted for $13.4 \%$, and Function 3 accounted for $6.9 \%$ (Table 4). Function 1 was most highly correlated with DA and E, while Function 2 was mostly highly correlated with Tb.N and Tb.Th (Table 6). Of the 49 individuals used to assess TCJ trabecular fabric properties, $57.1 \%$ of the cross-validated grouped cases were correctly classified (Table 7). Eighty percent of H. sapiens were classified correctly, while only $61 \%$ of P. troglodytes and 50\% of G. gorilla were classified correctly (Table 7). Gorilla gorilla were most often misclassified as $H$. sapiens (30\%), while G. beringei were most often misclassified as $P$. troglodytes (50\%).

TCJ VOI: StW 352 While BV/TV of StW 352 was closest to the P. troglodytes, G. gorilla, and G. beringei means, it was still just above the middle of the H. sapiens range (Fig. 4, Table 8). In 
StW 352, Tb.Th was higher than each extant group mean and fell only within the upper end of the P. troglodytes and G. gorilla range (Fig. 4, Table 8). In StW 352, Tb.N was lower than each extant group mean, falling within the lower end of the H. sapiens, P. troglodytes, and G. gorilla ranges, but below the G. beringei range (Fig. 4, Table 8). Thus, StW 352 has a relatively high $\mathrm{BV} / \mathrm{TV}$ in this VOI because of particularly thick trabecular struts, though they are few in number. This pattern is closest to the G. gorilla pattern, which pairs the highest mean Tb.Th with the lowest mean Tb.N amongst extant groups. In StW 352, DA was intermediate between $H$. sapiens and non-human group means, falling just below the middle of the $\mathrm{H}$. sapiens range, at the upper ends of the $P$. troglodytes and G. gorilla ranges, and above the upper end of the $G$. beringei range (Fig. 4, Table 8). In StW 352, E was higher than each extant group mean, falling within the upper ends of $H$. sapiens, $P$. troglodytes, and G. gorilla ranges, but above the $G$. beringei range (Table 8 ). Thus, relative to the extant groups, StW 352 had relatively anisotropic and rod-like trabecular struts. Primary trabecular orientation of StW 352 was A-P, as in extant groups, and fell within the $95 \%$ confidence intervals of $P$. troglodytes and G. gorilla, but outside the $95 \%$ confidence intervals of $H$. sapiens and G. beringei (Fig. 5). In the discriminant function analysis for the TCJ VOI, StW 352 had a predicted probability of being most similar to $G$. gorilla (0.727; Table 7).

\section{Discussion}

We tested the hypothesis that habitual joint forces acting on the calcaneus during the push-off phase of the gait cycle are preserved in trabecular architecture deep to the TCJ and CCJ, and that the morphology will be consistent with the predicted loading of each joint in each 
species. For the TCJ, we specifically hypothesized that, compared to P. troglodytes, G. gorilla, G. beringei, and P. ursinus, H. sapiens would have the most anisotropic and rod-like trabecular struts oriented in line with the predicted principal loads (i.e., in the A-P plane). More anisotropic struts in $H$. sapiens would appear to indicate less variable and more evenly distributed compressive loading during close-packing of the TCJ in preparation for a powerful push-off. In P. troglodytes, G. gorilla, and P. ursinus, which are thought to lack close-packing of the TCJ, we predicted more multidirectional loading of the subchondral surface. We find support for these hypotheses, with H. sapiens having the most anisotropic and rod-like trabecular struts at the TCJ, followed by G. gorilla (Table 9).

During push-off in $H$. sapiens and during the initial stages of the midtarsal break in $P$. troglodytes, G. gorilla, and Papio, the CCJ is thought to experience compressive forces through close-packing of this joint. In H. sapiens, both the dorsal and plantar aspects may experience equal loading with overall relatively low peak compressive forces (Nowak et al., 2010). However, in P. troglodytes, G. gorilla, G. beringei, and P. ursinus, only the dorsal aspect of the CCJ was predicted to show trabecular evidence of relatively high peak compressive loading (Nowak et al., 2010). Results for this joint provide only partial support for these predictions. Results for P. troglodytes provide support in having significantly higher Tb.Th and Tb.N in the dorsal compared to the plantar region of the CCJ. Contrary to predictions, however, H. sapiens also exhibited significantly higher Tb.Th, Tb.N, and BV/TV in the dorsal compared to the plantar region of the CCJ. If differentials in these properties between dorsal and plantar VOIs are in fact skeletal correlates of a midtarsal break, these unexpected results could indicate that the degree of midtarsal break variability exhibited in the H. sapiens $\mathrm{CCJ}$ is under-appreciated, as recent experimental work has suggested (Bates et al., 2013; DeSilva et al., 2015). It is worth 
noting, however, that the relatively high DA and E in dorsal and plantar regions of the H. sapiens CCJ are consistent with predictions of less variable, lower magnitude forces in a close-packed CCJ joint. Taking this mixed support into account, it is clear that loading patterns inferred from CCJ trabecular properties observed here differ from the loading patterns inferred from CCJ subchondral bone apparent density patterns of $H$. sapiens, $P$. troglodytes, and $P$. ursinus documented by Nowak et al. (2010).

The cause (or causes) of discrepant signals in the CCJ is unclear. One possibility is that subchondral and trabecular properties may not necessarily represent the same or even complementary biomechanical signals. For example, correlations of trabecular bone architecture and subchondral bone radiodensity patterns have been shown to be weak at the tibiotalar joint (distal tibia and talar trochlea) in humans, great apes, and baboons ( $\mathrm{Su}, 2011) . \mathrm{Su}(2011)$ suggested that each bone type responds to a specific form of loading: subchondral bone responds to loading of the complementary joint surface, while trabecular bone responds to forces traveling through the bone in question. Zeininger et al. (2011) also reported that subchondral bone in metacarpal heads may be more sensitive to external loading and show evidence of more recent modeling than deeper trabecular struts. Thus, a lack of correspondence at the CCJ between subchondral (Nowak et al., 2010) and trabecular bone properties (this study) is not necessarily contradictory, or even surprising. Future investigation of this issue at the CCJ should be extended to include future analyses of subchondral and trabecular bone at the complementary joint surface of the cuboid.

Despite the discrepant functional signals observed in trabecular properties deep to the $\mathrm{CCJ}$, it is worth noting that pairs of trabecular properties tended to regularly group together in explaining discriminant functions. Often, Tb.Th and Tb.N were paired and explained trends in 
$\mathrm{BV} / \mathrm{TV}$ amongst sample groups. Also, E and DA were frequently paired in explaining organizational trends in trabecular strut shape and orientation. Thus, our results support the findings of other studies (e.g., Ryan and Shaw, 2012; Barak et al., 2013; Scherf et al., 2015) in documenting the importance of considering suites of trabecular properties in VOIs rather than isolated properties. These pairings may be adaptive strategies for addressing functional requirements and future studies using experimental approaches are needed to explore their interdependency.

In each VOI examined here, $H$. sapiens usually had significantly lower BV/TV than other extant groups (Table 3). These results are consistent with a recent study reporting systemically lower $\mathrm{BV} / \mathrm{TV}$ in the human postcranial skeleton compared to non-human primates and preHolocene hominins (Chirchir et al., 2015). But a non-significant difference between H. sapiens and G. beringei, or between H. sapiens and P. ursinus in the dorsal CCJ VOI, is also consistent with the suggestion by Ryan and Shaw (2015) that human BV/TV may not always be systemically reduced in certain human populations (i.e., variability in modern human populations exists, possibility related to activity differences). Group differences in BV/TV are a function of two additional variables contributing to this metric: Tb.Th and Tb. N. Gorilla gorilla tends to achieve high BV/TV through having thicker, sparser struts. $P$. troglodytes and $P$. ursinus tend to achieve high BV/TV through having thinner, more numerous struts. These results, at least amongst extant taxa, may represent a body mass trend. Similar patterns of Tb.Th and $\mathrm{Tb}$. $\mathrm{N}$ have been reported for primate humeral and femoral heads (Ryan and Shaw, 2013), and in nonprimate mammals (Barak et al., 2013) in which larger individuals have an overall higher BV/TV comprised of few but thick struts, while smaller individuals have an overall lower BV/TV with more but thin struts. As noted above, H. sapiens has a consistently high DA throughout the 
calcaneus. Low BV/TV coupled with a high DA (i.e., relatively few, highly-oriented trabecular struts) has also been observed in the human femur (Ryan and Shaw, 2015) and metatarsal heads (Griffin et al., 2010). In human metatarsal heads, it has been suggested to be a functional strategy for mitigating compressive forces (i.e., at the metatarsophalangeal joint; Griffin et al., 2010), and results presented here also support this hypothesized functional strategy.

The pattern of trabecular properties in the StW 352 calcaneus is not consistent with any one extant group studied here. At the TCJ, StW 352 is most similar to G. gorilla in strut structure (fewer, thicker struts), but it resembles H. sapiens and G. gorilla in strut shape (rod-like) and strut orientation (relatively anisotropic; Table 9). This might suggest relatively less mobility in the TCJ of StW 352 (and by extension A. africanus) than in P. troglodytes. This is supported by the multivariate discriminant function analysis in which StW 352 TCJ trabeculae group with $G$. gorilla and $H$. sapiens, suggesting relatively less variable loading, possible close-packing of the TCJ (human-like), and a rigid foot during the stance phase of walking. Results presented here are consistent with those for the histomorphometrics of the StW 358 and StW 389 distal tibia trabeculae, in that A. africanus gait was inferred to be closest to humans but not entirely humanlike in these specimens (Barak et al., 2013). In a recent comparative study of the shape and orientation of the posterior talar articular facet, Prang (2016) reported that StW 352 was more similar to apes than humans and that this may be indicative of a more mobile TCJ in $A$. africanus. More details about the arthrokinematics of the TCJ in humans and non-human primates, particularly gorillas, using a method such as cineradiography are needed to make better predictions of habitual load-bearing, including possible regional differences in fossil hominins. Future studies examining shod versus unshod walking and walking on mountainous (i.e., 
uneven) versus flat terrain would provide further insight into the range of variability present in human pedal arthokinematics.

At the CCJ, StW 352 also falls between humans and African apes, aligning with $G$. beringei, G. gorilla, or with H. sapiens when trabecular properties of dorsal and plantar VOIs are considered separately (Table 9). At the CCJ, StW 352 is most similar to G. gorilla in strut structure (fewer, thicker struts), but resembles $P$. troglodytes or G. gorilla in strut orientation (relatively isotropic). In strut shape, StW 352 shows a similar pattern to that of $H$. sapiens and $P$. ursinus, in which struts are more rod-like in the dorsal VOI compared to the plantar VOI (Fig. 4). When considered together in a multivariate discriminant function analysis, trabeculae in the StW 352 dorsal CCJ VOI group with $P$. ursinus or H. sapiens, while trabeculae in the plantar CCJ VOI group with G. beringei or G. gorilla. Functionally, these results suggest overall postures and loading regimes that are not completely human-like or ape-like.

Recent analysis of the StW 352 calcaneal tuber external dimensions concluded that this fossil fell within the ape range, and particularly close to P. troglodytes (Prang, 2015). In the present study, StW 352 calcaneal trabeculae were observed to be not very Pan-like. Trabecular similarities between $\mathrm{StW} 352, H$. sapiens, G. gorilla, G. beringei, and P. ursinus (to the exclusion of $P$. troglodytes) document a clear indication of the relative importance of selection for terrestriality in the taxon represented by StW 352. Accordingly, anatomical structure based on internal trabecular architecture differs from external calcaneal anatomy documented by Prang (2015; i.e., calcaneal tuber robusticity). In considering this apparent discrepancy, it must be noted that the external assessment for an incomplete calcaneal tuber (Prang, 2015) may provide a different signal than if the entire tuber was present. As emphasized above, cineradiographic analyses of the Gorilla CCJ during midfoot break are needed to understand if/how Pan and 
Gorilla differ in this respect. With such data available, reinterpretation of the loading patterns of StW 352 would be greatly facilitated.

\section{Conclusion}

Given the lack of detailed data on G. gorilla or G. beringei CCJ kinematics, our trabecular results neither support nor refute the presence of calcaneocuboid joint flexion or a midtarsal break in StW 352. But at present, the CCJ of StW 352 morphologically appears to share Homo-like, Gorilla-like, and Papio-like features, all of which habitually use terrestrial substrates. Trabecular results for the TCJ suggest that StW 352 may have had a Homo-like, close-packed morphology in this joint. These findings suggest that the StW 352 calcaneus experienced more variable load orientations than $H$. sapiens calcanei, but usually less variable load orientations than $P$. troglodytes calcanei, the most arboreal taxon of our comparative sample. This conclusion suggests that A. africanus was capable of a large range of foot movements across its locomotor repertoire and this range differed from those exhibited by either humans or African apes.

\section{Acknowledgments}

We thank the following for providing much of the funding for this research: The Leakey Foundation (to AZ), the National Science Foundation (grant numbers: BCS 1028958 [to AZ] and BCS 1317047 [to BAP]), the Centre of Excellence-Palaeosciences (CoE-Pal) at the University of the Witwatersrand (to KJC), the National Research Foundation of South Africa (to KJC), and 
the Department of Science and Technology of South Africa (to KJC). Caley Orr and Midwestern University (Downers Grove, IL) also provided additional financial support for CT scanning the African ape specimens at the University of Southern California. We are grateful to the Fossil Access Advisory Committee of the University of the Witwatersrand for granting access to fossil specimen StW 352. We are also grateful to the Microfocus X-ray Computed Tomography (CT) Facility of the Palaeosciences Centre at the University of the Witwatersrand for providing access to their scanning facilities (www.wits.ac.za/microCT). The following individuals facilitated CT scanning at the Penn State University (http://www.cqi.psu.edu/): George Milner, Timothy Ryan, and Colin Shaw. The following individuals facilitated CT scanning at the University of TexasAustin (www.ctlab.geo.utexas.edu): Matt Colbert and Richard Ketcham. The following individuals facilitated CT scanning at the University of the Witwatersrand: Brendon Billings and Kudakwashe Jakata. The following individuals facilitated CT scanning at the University of Southern California (http://mic.usc.edu): Tautis Skorka, Bino Varghese, and Grant Dagliyan. We are indebted to the following individuals who provided access to specimens in their care and facilitated specimen loans: Brendon Billings, Neil Duncan, Nicole Edmison, Kristofer Helgen, Darrin Lunde, Terrance Martin, George Milner, and Eileen Westwig. We thank Anne Su for discussions concerning this project and its results. Finally, we thank the Editor, Associate Editor, and three anonymous reviewers for their constructive comments. 


\section{References}

Barak, M.M., Lieberman, D.E., Hublin, J., 2011. A Wolff in sheep's clothing: Trabecular bone adaptation in response to changes in joint loading orientation. Bone 49, 1141-1151.

Barak, M.M., Lieberman, D.E., Raichlen, D., Pontzer, H., Warrener, A.G., Hublin, J.J., 2013. Trabecular evidence for a human-like gait in Australopithecus africanus. PLOS One DOI, 10.1371/journal.pone.0077687.

Bates, K.T., Collins, D., Savage, R., McClymont, J., Webster, E., Pataky, T.C., D’Aout, K., Sellers, W.I., Bennett, M.R., Crompton, R.H., 2015. The evolution of compliance in the human lateral mid-foot. Proc. Royal Soc. B 280, 20131818.

Bojsen-Moller, F., 1979. Calcaneocuboid joint and stability of longitudinal arch of the foot at high and low gear push off. J. Anat. 129, 165-176.

Carlson, K.J., Patel, B.A., 2006. Habitual use of the primate forelimb is reflected in the material properties of subchondral bone in the distal radius. J. Anat. 208, 659-670.

Carlson, K.J., Jashashvili, T., Houghton, K., Westaway, M.C., Patel, B.A., 2013. Joint loads in marsupial ankles reflect habitual bipedalism versus quadrupedalism. PLoS One 8(3), e58811. 
Clarke, R.J., 2013. Australopithecus from Sterkfontein Caves, South Africa. In: Reed, K.E., Fleagle, J.G., Leakey, R.E. (Eds.), The Paleobiology of Australopithecus. Springer, New York. pp. $105-123$.

Chirchir, H., Kivell, T.L., Ruff, C.B., Hublin, J-J., Carlson, K.J., Zipfel, B., Richmond, B.G., 2015. Recent origin of low trabecular bone density in modern humans. Proc. Nat. Acad. Sci. USA $112,366-371$.

Close, J.R., Inman, V.T., Poor, P.M., Todd, F.N., 1967. The function of the subtalar joint. Clin. Ortho. Res. 15, 159-179.

Currey, J., 1984. The Mechanical Adaptations of Bone. Princeton University Press, Princeton.

D’Août, K., Aerts, P., De Clercq, D., De Meester, K., Van Elsacker, L., 2002. Segment and joint angles of hind limb during bipedal and quadrupedal walking of the bonobo (Pan paniscus). Am. J. Phys. Anthropol. 119, 37-51.

Deloison, Y., 2003. Fossil foot bone anatomy from South Africa between 2.4 and 3.5 Ma old. Interpretation to the kind of locomotion (trans). Biom. Hum. Et. Anthropol. 21, 189-230.

Deloison, Y., 2004. A new hypothesis on the origin of hominoid locomotion. In: Meldrum, J.D., Hilton, C.E. (Eds.), From Biped to Strider. Springer, New York. pp. 35-47. 
DeSilva, J.M., 2010. Revisiting the “midtarsal break.” Am. J. Phys. Anthropol. 141, 245-258.

DeSilva, J.M., Devlin, M., 2012. A comparative study of the trabecular bony architecture of the talus in non-human primates, and Australopithecus. J. Hum. Evol. 63, 536-551.

DeSilva, J.M., Gill, S.V., 2013. Brief communication: a midtarsal (midfoot) break in the human foot. Am. J. Phys. Anthropol. 151, 495-499.

DeSilva, J.M., Bonne-Annee, R., Swanson, Z., Gill, C.M., Sobel, M., Uy, J., Gill, S.V., 2015. Midtarsal break variation in modern humans: functional causes, skeletal correlates, and paleontological implications. Am. J. Phys. Anthropol. 156, 543-552.

Ding, M., Odgaard, A., Linde, F., Hvid, I., 2002. Age-related variation in the microstructure of human tibial cancellous bone. J. Orthop. Res. 20, 615-621.

Dunn, R.H., Tocheri, M.W., Orr, C.M., Jungers, W.L., 2014. Ecological divergence and talar morphology in gorillas. Am. J. Phys. Anthropol. 153, 526-541.

Elftman, H., Manter, J.T., 1935a. The evolution of the human foot, with especial reference to the joints. J. Anat. 70, 56-67.

Elftman, H., Manter, J.T., 1935b. Chimpanzee and human feet in bipedal walking. Am. J. Phys. Anthropol. 20, 69-79. 
Fajardo, R.J., Muller, R., 2001. Three-dimensional analysis of nonhuman primate trabecular bone architecture using micro-computed tomography. Am. J. Phys. Anthropol. 115, 827-886.

Giddings, V.L., Beaupre, G.S., Whalen, R.T., Carter, D.R., 2000. Calcaneal loading during walking and running. Med. Sci. Sports Exerc. 32, 627-634.

Goldstein, S.A., Matthews, L.S., Kuhn, J.L., Hollister, S.J., 1991. Trabecular bone remodeling: an experimental model. J. Biomech. 24, 35-150.

Griffin, N.L., D’Aout, K., Ryan, T.M., Richmond, B.G., Ketcham, R.A., Postnov, A., 2010. Comparative forefoot trabecular bone architecture in extant hominids. J. Hum. Evol. 59, 202213.

Hammer, O., Harper, D.A.T., Ryan, P.D., 2001. PAST: Paleontological Statistics Software Package for Education and Data Analysis: Paleontologica Electronica 4(1), 1-9.

Harrigan, T.P., Mann, R.W., 1984. Characterization of microstructural anisotropy in orthotropic materials using a second rank tensor. J. Mat. Sci. 19, 761-767.

Hodgskinson, R., Currey, J.D., 1990. The effect of variation in structure on the Young's modulus of cancellous bone: a comparison of human and non-human material. Proceedings of the Institution of Mechanical Engineers, Part H: J. Eng. Med. 204,115-121. 
Hoffler, C.E., Moore, K.E., Kozloff, K., Zysset, P.K., Brown, M.B., Goldstein, S.A., 2000.

Heterogeneity of bone lamellar-level elastic moduli. Bone 26, 603-609.

Kabel, J., van Rietergergen, B., Dalstra, M., Odgaard, A., Huiskes, R., 1999. The role of an effective isotropic tissue modulus in the elastic properties of cancellous bone. J. Biomech. 32, $673-680$.

Ketcham, R.A., Ryan, T.M., 2004. Quantification and visualization of anisotropy in trabecular bone. J. Microsc. 213(Pt 2), 158-171.

Kivell, T.L., Skinner, M.M., Lazenby, R., Hublin, J.J., 2011. Methodological considerations for analyzing trabecular architecture: an example from the primate hand. J. Anat. 218, 209-225.

Kuo, S., Desilva, J.M., Devlin, M.J., Mcdonald, G., Morgan, E.F., 2013. The effect of the Achilles tendon on trabecular structure in the primate calcaneus. Anat. Rec. 296, 1509-1517.

Langdon, J.H., Bruckner, J., Baker, H.H., 1991. Pedal mechanics and bipedalism in early hominids. In Origine(s) de la Bipedie chez les Hominides, Editions du CNRS, Paris.

Latimer, B., Lovejoy, C.O., 1989. The calcaneus of Australopithecus afarensis and its implications for the evolution of bipedality. Am. J. Phys. Anthropol. 78, 369-386. 
Lazenby, R.A., Skinner, M.M., Hublin, J.J., Boesch, C., 2011. Metacarpal trabecular architecture variation in the chimpanzee (Pan troglodytes): evidence for locomotion and tool-use? Am. J. Phys. Anthropol. 144, 215-225.

Leland, R.H., Marymont, J.V., Tervino, S.G., Varner, K.E., Noble, P.C., 2001. Calcaneocuboid stability: A clinical and anatomical study. Foot Ankle Int. 22, 880-884.

Lewis, O.J., 1980. The joints of the evolving foot. Part II. The intrinsic joints. J. Anat. 130, 833857.

MacConaill, M.A., Basmajian, J.V., 1969. Muscles and Movements. The Williams and Wilkins Company, Baltimore.

Maga, M., Kappelman, J., Ryan, T.M., Ketcham, R.A., 2006. Preliminary observations on the calcaneal trabecular microarchitecture of extant large-bodied hominoids. Am. J. Phys. Anthropol. 129, 410-417.

Meyer, G.H., 1867. Die Architektur der Spongiosa. Anat. Physiol. Wiss. Med. 34, 615-628.

Milner, G.R., Smith, V.G., 1990. Oneota human skeletal remains. In: Santure, S.K., Harn, A.D., Esarey, D. (Eds.), Archaeological Investigations at the Morton Village and Norris Farms 36 Cemetery. Illinois State Museum, Springfield, pp. 111-148. 
Morton, D.J., 1935. The human foot. Its evolution, physiology and functional disorders. Columbia University Press, New York.

Nowak, M.G., Carlson, K.J., Patel, B.A., 2010. Apparent density of the primate calcneo-cuboid joint and its association with locomotor mode, foot posture, and the "midtarsal break." Am. J. Phys. Anthropol. 142, 180-193.

Odgaard, A., 1997. Three-dimensional methods for quantification of cancellous bone architecture. Bone 20, 315-328.

Ouzounian, T.J., Shereff, M.J., 1989. In vitro determination of midfoot motion. Foot Ankle Int. $10,140-146$.

Patel, B.A., Carlson, K.J., 2007. Bone density spatial patterns in the distal radius reflect habitual hand postures adopted by quadrupedal primates. J. Hum. Evol. 52, 130-141.

Patel, B.A., Carlson, K.J., 2008. Apparent density patterns in subchondral bone of the sloth and anteater forelimb. Biol. Lett. 4, 486-489.

Polk, J.D., Blumefeld, J., Ahluwalia, K., 2008. Knee posture predicted from subchondral apparent density in the distal femur: an experimental validation. Anat. Rec. 291, 293-302.

Pontzer, H., Lieberman, D.E., Momin, E., Devlin, M.J., Polk, J.D., Hallgrimsson, B., 
Cooper, D.M.L., 2006. Trabecular bone in the bird knee responds with high sensitivity to changes in load orientation. J. Exp. Biol. 209, 57-65.

Prang, T.C., 2015. Calcaneal robusticity in Plio-Pleistocene hominins: Implications for locomotor diversity and phylogeny. J. Hum. Evol. 80, 135-146.

Prang, T.C., 2016. The subtalar joint complex of Australopithecus sediba. J. Hum. Evol. 90, $105-119$.

Rasband, W.S., 1997-2014. ImageJ, U.S. National Institutes of Health, Bethesda, Maryland, USA, http://imagej.nih.gov/ij/.

Ridler, T.W., Calvard, S., 1978. Picture thresholding using an iterative selection methods. IEEE Trans Syst Man Cybern SMC-8, 630-632.

Ryan, T.M., Shaw, C.N., 2012. Unique suites of trabecular bone features characterize locomotor behavior in human and non-human anthropoid primates. PLoS One 7(7), e41037.

Ryan, T.M., Shaw, C.N., 2013. Trabecular bone microstructure scales allometrically in the primate humerus and femur. Proc. Royal Soc. Lond. B 280, 20130172.

Ryan, T.M., Shaw, C.N., 2015. Gracility of the modern Homo sapiens skeleton is the result of decreased biomechanical loading. Proc. Nat. Acad. Sci. USA 112, 372-377. 
Ryan, T.M., Walker, A., 2010. Trabecular bone structure in the humeral and femoral heads of anthropoid primates. Anat. Rec. 293, 719-729.

Sammarco, V.J., 2004. The talonavicular and calcaneocuboid joints: anatomy, biomechanics, and clinical management of the transverse tarsal joint. Foot Ankle Clin. N. Am. 9, 127-145.

Sarrafian, S.K., 1993. Biomechanics of the subtalar joint complex. Clin. Ortho. Res. 290, 17-26.

Scherf, H., Wahl, J., Hublin, J.J., Havarti, K., 2015. Patterns of activity adaptation in humeral trabecular bone in Neolithic humans and present-day people. Am. J. Phys. Anthropol. 159, 106115.

Shea, B.T., 1981. Relative growth of the limbs and trunk in the African apes. Am. J. Phys. Anthropol. 56, 179-201.

Su, A., 2011. The functional morphology of subchondral and trabecular bone in the hominoid tibiotalar joint. PhD Dissertation, Stony Brook University.

Su, A., Carlson, K.J., in review. Comparative analysis of trabecular bone structure and orientation in South African hominin tali. J. Hum. Evol. 
Su, A., Wallace, I. J., Nakatsukasa, M., 2013. Trabecular bone anisotropy and orientation in an Early Pleistocene hominin talus from East Turkana, Kenya. J. Hum. Evol. 64, 667-677.

Susman, R.L., 1983. Evolution of the human foot: evidence from Plio-Pleistocene hominids. Foot Ankle 3, 365-376.

Thompson, N.E., Holowka, N.B., O'Neill, M.C., Larson, S.G., 2014. Brief communication: cineradiographic analysis of the chimpanzee (Pan troglodytes) talonavicular and calcaneocuboid joints. Am. J. Phys. Anthropl. 154, 604-608.

Tocheri, M.W., Solhan, C.R., Orr, C.M., Fermiani, J., Frolich, B., Groves, C.P., Harcourt-Smith, W.E., Richmond, B.G., Shoelson, B., Jungers, W.L. 2011. Ecological divergence and medial cuneiform morphology in gorillas. J. Hum. Evol. 60, 171-184.

Trussel, H.J., 1979. Comments on "Picture thresholding using an iterative selection method." IEEE Trans. Syst. Man. Cybern SMC 9, 311.

Turner, C.H., Coin, S.C., Rho, J.Y., Ashman, R.B., Rice, J.C., 1990. The fabric dependence of the orthotropic elastic constants of cancellous bone. J. Biomech. 23, 549-561.

Ulrich, D., van Rietbergen, B., Laib, A., Ruegsegger, P., 1999. The ability of three-dimensional structural indices to reflect mechanical aspects of trabecular bone. Bone 25, 55-60. 
Vereecke, E., D’Août, K., De Clercq, D., Van Elsacker, L., Aerts, P., 2003. Dynamic plantar pressure distribution during terrestrial locomotion of bonobos (Pan paniscus). Am. J. Phys. Anthropol. 120, 373-383.

Wolff, J., 1892. Das Gesetz der Transformation der Knochen. A. Hirchwild, Berlin.

Zeininger, A., Richmond, B.G., Hartman, G., 2011. Metacarpal head biomechanics: A comparative backscattered electron image analysis of trabecular bone mineral density in Pan troglodytes, Pongo pygmaeus, and Homo sapiens. J. Hum. Evol. 60, 703-710.

Zipfel, B., DeSilva, J.M., Kidd, R.S., Carlson, K.J., Churchill, S.E., Berger, L.R., 2011. The foot and ankle of Australopithecus sediba. Science 333, 1417-1420 


\section{Figure legends}

Figure 1. Photographs of the StW 352 partial right calcaneus (top) and images of a rendering (bottom) generated from high resolution CT scans. Left = lateral view, right $=$ anterior view.

Figure 2. Predicted joint reaction forces. In the human (left), the talocalcaneal joint (TCJ) and calcaneocuboid joint (CCJ; dorsal and plantar aspects) experience compressive force (black arrows) during the push-off phase of stance. In P. troglodytes (right), G. gorilla, and P. ursinus, the dorsal aspect of the CCJ presumably experiences compressive force (black arrow) during midfoot flexion.

Figure 3. Volume of interest (VOI) locations. Sagittal cross sections through microCT scans of (a) P. ursinus, (b) P. troglodytes, (c) StW 352, (d) G. gorilla, and (e) H. sapiens calcanei. White circles indicate VOI locations. For illustrative purposes, cubic representations of trabecular structure are provided for each VOI. Actual quantification of trabecular properties was performed on spherical VOIs.

Figure 4. Box plots of trabecular fabric properties for each volume of interest. Boxes envelop the inter-quartile range (50\% of values) of the sample distribution, and whiskers encompass the range excluding outliers, which are represented with open circles and stars. The horizontal line 
within each box illustrates the median of the distribution. H.s. $=$ Homo sapiens, P.t. $=$ Pan troglodytes, G.g. $=$ Gorilla gorilla, G.b. $=$ Gorilla beringei, and P.u. $=$ Papio ursinus.

Figure 5. Stereoplots of primary trabecular orientation, where top $=$ dorsal CCJ, middle $=$ plantar $\mathrm{CCJ}$, and bottom $=\mathrm{TCJ}$. In order to interpret this information, individuals falling toward the top and bottom poles have a primary trabecular orientation in the dorsoplantar plane. Individuals located to the left and right have a mediolateral primary trabecular orientation. The anteroposterior (A-P) plane runs through the middle of the sphere such that individuals located toward the center of the plot have an A-P primary trabecular orientation. Ellipses indicate the $95 \%$ confidence intervals for groups. 
Table 1. Sample sizes and diameters of volumes of interest. ${ }^{\mathrm{a}}$

\begin{tabular}{lccccccc}
\hline & \multicolumn{3}{c}{ TCJ } & \multicolumn{3}{c}{ CCJ $^{\mathbf{b}}$} \\
\hline Taxon & $\boldsymbol{n}$ & Mean $(\mathbf{m m})$ & Range & sd & Mean (mm) & Range & sd \\
StW 352 & 1 & 10.96 & - & - & 4.54 & - & - \\
H. sapiens & 15 & 12.29 & $9.34-15.00$ & 1.91 & 7.59 & $6.12-9.25$ & 0.93 \\
$P$. troglodytes & 18 & 10.32 & $7.67-12.19$ & 1.35 & 5.86 & $4.77-6.80$ & 0.64 \\
G. gorilla & 10 & 13.03 & $9.80-16.15$ & 2.28 & 7.52 & $5.88-9.45$ & 1.25 \\
G. beringei & 6 & 12.57 & $9.62-14.68$ & 2.14 & 7.38 & $5.87-8.27$ & 1.01 \\
$P$. ursinus & 11 & - & - & - & 3.82 & $3.28-4.26$ & 0.30 \\
\hline TCJ & & & & & & & \\
\hline
\end{tabular}

${ }^{\mathrm{a}} \mathrm{TCJ}=$ talocalcaneal joint, $\mathrm{CCJ}=$ calaneocuboid joint

${ }^{\mathrm{b}}$ Equivalently-sized dorsal and plantar volumes of interest were used in a given specimen. 


\begin{tabular}{|c|c|c|c|c|c|c|}
\hline & Taxon & DA & $\mathbf{E}$ & BV/TV & Tb.Th & Tb.N \\
\hline \multirow[t]{16}{*}{ Dorsal CCJ } & StW 352 & 0.78 & 0.71 & 0.26 & 0.21 & 1.07 \\
\hline & H. sapiens & 0.92 & 0.87 & 0.29 & 0.19 & 1.34 \\
\hline & & $(0.02)$ & $(0.04)$ & $(0.04)$ & $(0.03)$ & $(0.16)$ \\
\hline & & $0.88-0.94$ & $0.78-0.92$ & $0.23-0.36$ & $0.14-0.23$ & $1.12-1.77$ \\
\hline & P. troglodytes & 0.73 & 0.48 & 0.35 & 0.19 & 1.48 \\
\hline & & $(0.09)$ & $(0.20)$ & $(0.05)$ & $(0.04)$ & $(0 . .28)$ \\
\hline & & $0.57-0.84$ & $0.15-0.77$ & $0.29-0.45$ & $0.15-0.26$ & $0.97-1.92$ \\
\hline & G. gorilla & 0.81 & 0.50 & 0.37 & 0.24 & 1.25 \\
\hline & & $(0.07)$ & $(0.17)$ & $(0.08)$ & $(0.06)$ & $(0.23)$ \\
\hline & & $0.69-0.92$ & $0.26-0.78$ & $0.25-0.53$ & $0.19-0.39$ & $0.95-1.58$ \\
\hline & G. beringei & 0.78 & 0.45 & 0.34 & 0.22 & 1.25 \\
\hline & & $(0.09)$ & $(0.19)$ & $(0.08)$ & $(0.03)$ & $(0.11)$ \\
\hline & & $0.64-0.87$ & $0.25-0.74$ & $0.22-0.47$ & $0.18-0.28$ & $1.12-1.45$ \\
\hline & P. ursinus & 0.82 & 0.68 & 0.33 & 0.18 & 1.46 \\
\hline & & $(0.05)$ & $(0.11)$ & $(0.07)$ & $(0.02)$ & $(0.21)$ \\
\hline & & $0.74-0.89$ & $0.47-0.82$ & $0.21-0.43$ & $0.16-0.20$ & $1.08-1.71$ \\
\hline Plantar CCJ & StW 352 & 0.39 & 0.13 & 0.41 & 0.25 & 1.33 \\
\hline
\end{tabular}




$\begin{array}{lccccc}\text { H. sapiens } & 0.88 & 0.54 & 0.18 & 0.16 & 0.95 \\ & (0.06) & (0.19) & (0.06) & (0.03) & (0.25) \\ & 0.75-0.96 & 0.10-0.82 & 0.11-0.32 & 0.13-0.25 & 0.66-1.52 \\ \text { P. troglodytes } & 0.77 & 0.44 & 0.34 & 0.18 & 1.56 \\ & (0.08) & (0.15) & (0.05) & (0.04) & (0.30) \\ \text { G. gorilla } & 0.62-0.89 & 0.13-0.66 & 0.25-0.43 & 0.13-0.27 & 1.14-1.96 \\ & 0.74 & 0.48 & 0.36 & 0.22 & 1.34 \\ & (0.07) & (0.17) & (0.07) & (0.03) & (0.20) \\ \text { G. beringei } & 0.60-0.86 & 0.25-0.73 & 0.25-0.45 & 0.18-0.28 & 0.91-1.60 \\ & 0.72 & 0.41 & 0.31 & 0.21 & 1.28 \\ & (0.07) & (0.14) & (0.08) & (0.05) & (0.15) \\ & 0.63-0.81 & 0.22-0.54 & 0.22-0.41 & 0.15-0.30 & 1.08-1.47 \\ & 0.75 & 0.55 & 0.30 & 0.16 & 1.47 \\ & (0.10) & (0.15) & (0.03) & (0.02) & (0.08) \\ & 0.56-0.90 & 0.30-0.81 & 0.26-0.34 & 0.13-0.19 & 1.30-1.60\end{array}$

${ }^{\mathrm{a}} \mathrm{DA}=$ degree of anisotropy, $\mathrm{E}=$ elongation index, $\mathrm{BV} / \mathrm{TV}=$ bone volume fraction, $\mathrm{Tb} . \mathrm{Th}=$ trabecular thickness, $\mathrm{Tb} . \mathrm{N}=$ trabecular number. Cells report means, one standard deviation in parentheses below, and ranges at the bottom. 
Table 3: Kruskal-Wallis and pairwise Mann-Whitney U results for individual variables in calcaneocuboid (CCJ) and talocalcaneal (TCJ) volumes of interest. ${ }^{\mathrm{a}}$

\begin{tabular}{|c|c|c|c|c|c|c|}
\hline & & DA & $\mathbf{E}$ & BV/TV & Tb.Th & Tb.N \\
\hline \multicolumn{7}{|l|}{ Dorsal } \\
\hline \multirow[t]{11}{*}{$\mathrm{CCJ}$} & Kruskal-Wallis & $\mathrm{H}=37.24, \boldsymbol{p}<\mathbf{0 . 0 0 1}$ & $\mathrm{H}=38.34, \boldsymbol{p}<\mathbf{0 . 0 0 1}$ & $\mathrm{H}=10.13, \boldsymbol{p}=\mathbf{0 . 0 3 8}$ & $\mathrm{H}=15.55, \boldsymbol{p}=\mathbf{0 . 0 0 3}$ & $\mathrm{H}=9.55, \boldsymbol{p}=\mathbf{0 . 0 4 9}$ \\
\hline & H. sapiens vs. P. troglodytes & $<0.001$ & $<0.001$ & 0.001 & 0.899 & 0.164 \\
\hline & H. sapiens vs. G. gorilla & $<0.001$ & $<0.001$ & 0.025 & 0.005 & 0.437 \\
\hline & H. sapiens vs. G. beringei & $<0.001$ & $<0.001$ & 0.172 & 0.054 & 0.213 \\
\hline & H. sapiens vs. P. ursinus & $<0.001$ & $<0.001$ & 0.194 & 0.235 & 0.108 \\
\hline & P. troglodytes vs. G. gorilla & 0.022 & 0.829 & 0.665 & 0.033 & 0.039 \\
\hline & P. troglodytes vs. G. beringei & 0.229 & 0.789 & 0.763 & 0.169 & 0.039 \\
\hline & P. troglodytes vs. $P$. ursinus & 0.005 & 0.010 & 0.194 & 0.305 & 0.621 \\
\hline & G. gorilla vs. P. ursinus & 0.671 & 0.018 & 0.340 & $<0.001$ & 0.038 \\
\hline & G. beringei vs. $P$. ursinus & 0.512 & 0.014 & 0.999 & 0.007 & 0.068 \\
\hline & G. gorilla vs. G. beringei & 0.413 & 0.550 & 0.414 & 0.622 & 0.957 \\
\hline \multicolumn{7}{|c|}{ Plantar } \\
\hline \multirow[t]{3}{*}{ CCJ } & Kruskal-Wallis & $\mathrm{H}=23.85, \boldsymbol{p}<\mathbf{0 . 0 0 1}$ & $\mathrm{H}=6.023, p=0.197$ & $\mathrm{H}=32.69, \boldsymbol{p}<\mathbf{0 . 0 0 1}$ & $\mathrm{H}=19.27, \boldsymbol{p}<\mathbf{0 . 0 0 1}$ & $\mathrm{H}=28.35, \boldsymbol{p}<\mathbf{0 . 0 0 1}$ \\
\hline & H. sapiens vs. P. troglodytes & $<0.001$ & - & $<0.001$ & 0.077 & $<0.001$ \\
\hline & H. sapiens vs. G. gorilla & $<0.001$ & - & $<0.001$ & 0.001 & 0.002 \\
\hline
\end{tabular}




$\begin{array}{lllllr}\text { H. sapiens vs. G. beringei } & \mathbf{0 . 0 0 2} & - & \mathbf{0 . 0 0 4} & \mathbf{0 . 0 3 7} & \mathbf{0 . 0 1 1} \\ \text { H. sapiens vs. P. ursinus } & \mathbf{0 . 0 0 1} & - & <\mathbf{0 . 0 0 1} & 0.526 & <\mathbf{0 . 0 0 1} \\ \text { P. troglodytes vs. G. gorilla } & 0.157 & - & 0.682 & \mathbf{0 . 0 0 9} & 0.103 \\ \text { P. } \text { troglodytes vs. G. beringi } & 0.230 & - & 0.439 & 0.330 & 0.062 \\ \text { P. } \text { troglodytes vs. P. ursinus } & 0.528 & - & \mathbf{0 . 0 1 6} & 0.247 & 0.514 \\ \text { G. gorilla vs. P. ursinus } & 0.340 & - & \mathbf{0 . 0 4 7} & \mathbf{0 . 0 0 1} & 0.181 \\ \text { G. beringei vs. P. ursinus } & 0.615 & - & 0.449 & 0.076 & \mathbf{0 . 0 1 2} \\ \text { G. gorilla vs. G. beringei } & 0.664 & - & 0.383 & 0.586 & 0.385\end{array}$

\begin{tabular}{|c|c|c|c|c|c|c|}
\hline \multirow[t]{6}{*}{ TCJ } & Kruskal-Wallis & $\mathrm{H}=25.67, \boldsymbol{p}<\mathbf{0 . 0 0 1}$ & $\mathrm{H}=18.64, \boldsymbol{p}<\mathbf{0 . 0 0 1}$ & $\mathrm{H}=18.34, \boldsymbol{p}<\mathbf{0 . 0 0 1}$ & $\mathrm{H}=6.03, p=0.108$ & $\mathrm{H}=9.23, \boldsymbol{p}=\mathbf{0 . 0 2 6}$ \\
\hline & H. sapiens vs. P. troglodytes & $<0.001$ & $<0.001$ & $<0.001$ & - & 0.058 \\
\hline & H. sapiens vs. G. gorilla & 0.005 & 0.999 & 0.015 & - & 0.222 \\
\hline & H. sapiens vs. G. beringei & 0.002 & 0.006 & 0.035 & - & 0.846 \\
\hline & P. troglodytes vs. G. gorilla & 0.016 & 0.006 & 0.049 & - & 0.010 \\
\hline & G. gorilla vs. G. beringei & 0.039 & 0.026 & 0.703 & - & 0.064 \\
\hline
\end{tabular}

${ }^{\mathrm{a}} \mathrm{DA}=$ degree of anisotropy, $\mathrm{E}=$ elongation index, $\mathrm{BV} / \mathrm{TV}=$ bone volume fraction, $\mathrm{Tb} . \mathrm{Th}=$ trabecular thickness, Tb.N $=$ trabecular number. Values in bold indicate statistically significant results. 
Table 4. Outcome of discriminant function analyses.

\begin{tabular}{|c|c|c|c|c|c|}
\hline $\mathrm{VOI}^{\mathrm{a}}$ & Function & Eigenvalue & $\%$ of Variance & Cumulative \% & $\begin{array}{c}\text { Canonical } \\
\text { correlation }\end{array}$ \\
\hline \multirow[t]{4}{*}{ Dorsal CCJ } & 1 & 1.558 & 57.8 & 57.8 & 0.780 \\
\hline & 2 & 1.107 & 41.1 & 98.9 & 0.725 \\
\hline & 3 & 0.025 & 0.9 & 99.9 & 0.158 \\
\hline & 4 & 0.003 & 0.1 & 100.0 & 0.056 \\
\hline \multirow[t]{4}{*}{ Plantar CCJ } & 1 & 1.949 & 75.9 & 75.9 & 0.813 \\
\hline & 2 & 0.394 & 15.3 & 91.3 & 0.531 \\
\hline & 3 & 0.200 & 7.8 & 99.1 & 0.408 \\
\hline & 4 & 0.024 & 0.9 & 100.0 & 0.154 \\
\hline \multirow[t]{4}{*}{ Combined CCJ } & 1 & 3.010 & 64.1 & 64.1 & 0.866 \\
\hline & 2 & 1.204 & 25.7 & 89.8 & 0.739 \\
\hline & 3 & 0.411 & 8.8 & 98.6 & 0.540 \\
\hline & 4 & 0.068 & 1.4 & 100.0 & 0.252 \\
\hline \multirow[t]{3}{*}{ TCJ } & 1 & 1.315 & 79.8 & 79.8 & 0.754 \\
\hline & 2 & 0.220 & 13.4 & 93.1 & 0.425 \\
\hline & 3 & 0.113 & 6.9 & 100.0 & 0.319 \\
\hline
\end{tabular}

${ }^{\mathrm{a}} \mathrm{CCJ}=$ calcaneocuboid joint, $\mathrm{TCJ}=$ talocalcaneal joint. 
Table 5. Structure coefficients of discriminant function analyses. ${ }^{a, b}$

\begin{tabular}{|c|c|c|c|c|c|}
\hline Dorsal CCJ & Variable & Function 1 & Function 2 & Function 3 & Function 4 \\
\hline & $\mathrm{E}$ & 0.892 & -0.251 & 0.353 & -0.127 \\
\hline & DA & 0.845 & 0.174 & 0.060 & 0.503 \\
\hline & Tb.N & -0.114 & -0.408 & 0.447 & 0.788 \\
\hline & Tb.Th & -0.164 & 0.530 & 0.548 & -0.626 \\
\hline \multirow[t]{5}{*}{ Plantar CCJ } & Variable & Function 1 & Function 2 & Function 3 & Function 4 \\
\hline & Tb.N & 0.709 & -0.609 & 0.263 & 0.238 \\
\hline & Tb.Th & 0.313 & 0.880 & -0.137 & 0.331 \\
\hline & E & -0.156 & -0.195 & -0.359 & 0.899 \\
\hline & DA & -0.519 & -0.119 & 0.576 & 0.620 \\
\hline \multirow[t]{9}{*}{ Combined CCJ } & Variable & Function 1 & Function 2 & Function 3 & Function 4 \\
\hline & Dorsal E & -0.636 & -0.115 & -0.406 & 0.409 \\
\hline & Dorsal DA & -0.571 & 0.286 & -0.381 & 0.326 \\
\hline & Plantar Tb.N & 0.559 & -0.383 & -0.196 & 0.355 \\
\hline & Dorsal Tb.Th & 0.140 & 0.471 & 0.270 & 0.280 \\
\hline & Plantar Tb.Th & 0.266 & 0.468 & 0.240 & 0.142 \\
\hline & Dorsal Tb.N & 0.058 & -0.404 & -0.048 & 0.238 \\
\hline & Plantar E & -0.135 & -0.020 & -0.308 & 0.442 \\
\hline & Plantar DA & -0.415 & -0.135 & 0.361 & 0.429 \\
\hline \multirow[t]{3}{*}{ TCJ } & Variable & Function 1 & Function 2 & Function 3 & \\
\hline & DA & -0.827 & 0.502 & 0.225 & \\
\hline & $\mathrm{E}$ & -0.619 & -0.152 & 0.419 & \\
\hline
\end{tabular}




$\begin{array}{cccc}\text { Tb.N } & 0.410 & 0.754 & 0.136 \\ \text { Tb.Th } & 0.201 & -0.573 & 0.525\end{array}$

${ }^{a}$ Pooled within-group correlations between discriminating variables and standardized canonical
discriminant functions.

${ }^{\mathrm{b}} \mathrm{CCJ}=$ calcaneocuboid joint, $\mathrm{TCJ}=$ talocalcaneal joint. 
Table 6. Classification results of discriminant function analyses. ${ }^{\text {a,b }}$

\begin{tabular}{|c|c|c|c|c|c|c|c|}
\hline $\mathrm{VOI}^{\mathrm{b}}$ & $\begin{array}{c}\text { Original } \\
\text { grouped cases } \\
\text { correctly } \\
\text { classified } \\
\text { (Cross- }\end{array}$ & $\begin{array}{c}\text { Original } \\
\text { group }\end{array}$ & Pre & icted group & nembers & p (actual/t & al) \\
\hline \multirow[t]{7}{*}{ Dorsal CCJ } & $63.3 \%(55.0 \%)$ & & $\begin{array}{c}H . \\
\text { sapiens }\end{array}$ & $\begin{array}{c}P . \\
\text { troglodytes }\end{array}$ & $\begin{array}{c}G . \\
\text { gorilla }\end{array}$ & $\begin{array}{c}G . \\
\text { beringei }\end{array}$ & $\begin{array}{c}P . \\
\text { ursinus }\end{array}$ \\
\hline & & Stw352 & 0.307 & & & & 0.469 \\
\hline & & H. sapiens & $14 / 15$ & $0 / 15$ & $0 / 15$ & $0 / 15$ & $1 / 15$ \\
\hline & & P. troglodytes & $0 / 18$ & $12 / 18$ & $1 / 18$ & $1 / 18$ & $4 / 18$ \\
\hline & & G. gorilla & $1 / 10$ & $2 / 10$ & $7 / 10$ & $0 / 10$ & $0 / 10$ \\
\hline & & G. beringei & $1 / 6$ & $1 / 6$ & $3 / 6$ & $1 / 6$ & $0 / 6$ \\
\hline & & P. ursinus & $3 / 11$ & $4 / 11$ & $0 / 11$ & $0 / 11$ & $4 / 11$ \\
\hline \multirow[t]{7}{*}{$\begin{array}{l}\text { Plantar } \\
\text { CCJ }\end{array}$} & $60.0 \%(55.0 \%)$ & & $\begin{array}{c}H . \\
\text { sapiens }\end{array}$ & $\begin{array}{c}P . \\
\text { troglodytes }\end{array}$ & $\begin{array}{c}\text { G. } \\
\text { gorilla }\end{array}$ & $\begin{array}{c}G . \\
\text { beringei }\end{array}$ & $\begin{array}{c}P . \\
\text { ursinus }\end{array}$ \\
\hline & & $\operatorname{Stw} 352^{\mathrm{a}}$ & & & 0.444 & 0.494 & \\
\hline & & H. sapiens & $12 / 15$ & $0 / 15$ & $1 / 15$ & $0 / 15$ & $2 / 15$ \\
\hline & & P. troglodytes & $1 / 18$ & $11 / 18$ & $2 / 18$ & $1 / 18$ & $3 / 18$ \\
\hline & & G. gorilla & $0 / 10$ & $2 / 10$ & $6 / 10$ & $0 / 10$ & $2 / 10$ \\
\hline & & G. beringei & $0 / 6$ & $2 / 6$ & $3 / 6$ & $0 / 6$ & $1 / 6$ \\
\hline & & P. ursinus & $1 / 11$ & $3 / 11$ & $0 / 11$ & $0 / 11$ & $7 / 11$ \\
\hline $\begin{array}{l}\text { Combined } \\
\text { CCJ }\end{array}$ & $73.3 \%(56.7 \%)$ & & $\begin{array}{c}H . \\
\text { sapiens }\end{array}$ & $\begin{array}{c}P . \\
\text { troglodytes }\end{array}$ & $\begin{array}{c}\text { G. } \\
\text { gorilla }\end{array}$ & $\begin{array}{c}G . \\
\text { beringei }\end{array}$ & $\begin{array}{c}P . \\
\text { ursinus }\end{array}$ \\
\hline
\end{tabular}




\begin{tabular}{|c|c|c|c|c|c|c|c|}
\hline \multicolumn{6}{|c|}{ Stw352 $2^{\mathrm{a}}$} & \multirow{2}{*}{$\begin{array}{l}0.352 \\
0 / 15\end{array}$} & \multirow{2}{*}{$\begin{array}{r}\mathbf{0 . 5 5 2} \\
1 / 15\end{array}$} \\
\hline & & H. sapiens & $14 / 15$ & $0 / 15$ & $0 / 15$ & & \\
\hline & & P. troglodytes & $2 / 18$ & $13 / 18$ & $2 / 18$ & $0 / 18$ & $1 / 18$ \\
\hline & & G. gorilla & $0 / 10$ & $2 / 10$ & $7 / 10$ & $0 / 10$ & $1 / 10$ \\
\hline & & G. beringei & $0 / 6$ & $1 / 6$ & $4 / 6$ & $1 / 6$ & $0 / 6$ \\
\hline & & P. ursinus & $0 / 11$ & $2 / 11$ & $0 / 11$ & $0 / 11$ & $9 / 11$ \\
\hline \multirow[t]{6}{*}{ TCJ } & $61.2 \%(57.1 \%)$ & & $\begin{array}{c}H . \\
\text { sapiens }\end{array}$ & $\begin{array}{c}P . \\
\text { troglodytes }\end{array}$ & $\begin{array}{c}G . \\
\text { gorilla }\end{array}$ & $\begin{array}{c}G . \\
\text { beringei }\end{array}$ & \\
\hline & & Stw352 & 0.152 & & 0.727 & & \\
\hline & & H. sapiens & $12 / 15$ & $2 / 15$ & $0 / 15$ & $1 / 15$ & \\
\hline & & P. troglodytes & $2 / 18$ & $11 / 18$ & $3 / 18$ & $2 / 18$ & \\
\hline & & G. gorilla & $3 / 10$ & $1 / 10$ & $5 / 10$ & $1 / 10$ & \\
\hline & & G. beringei & $1 / 6$ & $3 / 6$ & $0 / 6$ & $2 / 6$ & \\
\hline
\end{tabular}

${ }^{\mathrm{a}}$ First (in bold) and second highest classification probability values for Stw 352. Others not reported.

${ }^{\mathrm{b}} \mathrm{CCJ}=$ calcaneocuboid joint, $\mathrm{TCJ}=$ talocalcaneal joint. 
Table 7. Wilcoxon results for dorsal vs. plantar calcaneocuboid joint (CCJ) volumes of interest. ${ }^{a}$

\begin{tabular}{lccccc}
\hline & \multicolumn{5}{c}{ Dorsal vs. plantar CCJ } \\
\hline DA & $\mathbf{E}$ & $\mathbf{B V} / \mathbf{T V}$ & Tb.Th & Tb.N \\
H. sapiens & 0.051 & $<\mathbf{0 . 0 0 1}$ & $<\mathbf{0 . 0 0 1}$ & $\mathbf{0 . 0 0 8}$ & $<\mathbf{0 . 0 0 1}$ \\
P. troglodytes & 0.257 & 0.723 & 0.909 & $\mathbf{0 . 0 4 9}$ & $\mathbf{0 . 0 2 5}$ \\
G. gorilla & $\mathbf{0 . 0 4 1}$ & 0.953 & 0.645 & 0.201 & 0.074 \\
G. beringei & 0.075 & 0.600 & 0.685 & 0.346 & 0.346 \\
P. ursinus & $\mathbf{0 . 0 3 7}$ & $\mathbf{0 . 0 4 1}$ & 0.155 & 0.196 & 0.999
\end{tabular}

${ }^{\mathrm{a}} \mathrm{DA}=$ degree of anisotropy, $\mathrm{E}=$ elongation index, $\mathrm{BV} / \mathrm{TV}=$ bone volume fraction, $\mathrm{Tb} . \mathrm{Th}=$
trabecular thickness, $\mathrm{Tb} . \mathrm{N}=$ trabecular number. Values in bold indicate statistically significant
results. 
Table 8. Talocalcaneal joint (TCJ) volume of interest trabecular fabric properties., ${ }^{\mathrm{a} b}$

\begin{tabular}{|c|c|c|c|c|c|c|}
\hline & Taxon & DA & $\mathbf{E}$ & BV/TV & Tb.Th & Tb.N \\
\hline \multirow[t]{13}{*}{ TCJ } & StW 352 & 0.85 & 0.68 & 0.43 & 0.36 & 1.14 \\
\hline & H. sapiens & 0.91 & 0.66 & 0.35 & 0.25 & 1.28 \\
\hline & & $(0.06)$ & $(0.11)$ & $(0.06)$ & $(0.04)$ & $(0.14)$ \\
\hline & & $0.72-0.97$ & $0.41-0.81$ & $0.27-0.50$ & $0.18-0.33$ & $1.02-1.57$ \\
\hline & P. troglodytes & 0.74 & 0.49 & 0.45 & 0.28 & 1.46 \\
\hline & & $(0.09)$ & $(0.13)$ & $(0.05)$ & $(0.05)$ & $(0.25)$ \\
\hline & & $0.52-0.89$ & $0.20-0.68$ & $0.35-0.51$ & $0.21-0.39$ & $1.10-1.89$ \\
\hline & G. gorilla & 0.83 & 0.65 & 0.41 & 0.29 & 1.19 \\
\hline & & $(0.07)$ & $(0.14)$ & $(0.05)$ & $(0.04)$ & $(0.17)$ \\
\hline & & $0.70-0.90$ & $0.41-0.86$ & $0.37-0.50$ & $0.24-0.37$ & $1.04-1.39$ \\
\hline & G. beringei & 0.72 & 0.49 & 0.41 & 0.27 & 1.30 \\
\hline & & $(0.10)$ & $(0.09)$ & $(0.06)$ & $(0.03)$ & $(0.08)$ \\
\hline & & $0.58-0.83$ & $0.33-0.55$ & $0.33-0.50$ & $0.24-0.31$ & $1.23-1.45$ \\
\hline
\end{tabular}

${ }^{\mathrm{a}} \mathrm{DA}=$ degree of anisotropy, $\mathrm{E}=$ elongation index, $\mathrm{BV} / \mathrm{TV}=$ bone volume fraction, $\mathrm{Tb} . \mathrm{Th}=$ trabecular thickness, Tb. $\mathrm{N}=$ trabecular number. Cells report means, one standard deviation in parentheses below, and ranges at the bottom. 
Table 9. Results summary for trabecular fabric comparisons., ${ }^{\mathrm{a}, \mathrm{b}, \mathrm{c}}$

DA

\begin{tabular}{|c|c|c|c|c|c|}
\hline Dorsal CCJ & $\mathrm{H}>\mathrm{B}>\mathrm{Gg}>\mathrm{Gb}>\underline{\mathrm{StW}} 352>\mathrm{P}$ & $\mathrm{H}>\underline{\mathrm{StW}} 352>\mathrm{B}>\mathrm{Gg}>\mathrm{P}>\mathrm{Gb}$ & $\mathrm{Gg}>\mathrm{P}>\mathrm{Gb}>\mathrm{B}>\mathrm{H}>\underline{\mathrm{StW}} 352$ & $\mathrm{Gg}>\mathrm{Gb}>\underline{\mathrm{StW} 352}>\mathrm{P}>\mathrm{H}>\mathrm{B}$ & $\mathrm{P}>\mathrm{B}>\mathrm{H}>\mathrm{Gg}>\mathrm{Gb}>\underline{\mathrm{StW} 352}$ \\
\hline Plantar CCJ & $\mathrm{H}>\mathrm{P}>\mathrm{B}>\mathrm{Gg}>\mathrm{Gb}>\underline{\mathrm{StW} 352}$ & $\mathrm{~B}>\mathrm{H}>\mathrm{Gg}>\mathrm{P}>\mathrm{Gb}>\underline{\mathrm{StW}} 352$ & $\underline{\text { StW352 }}>\mathrm{Gg}>\mathrm{P}>\mathrm{Gb}>\mathrm{B}>\mathrm{H}$ & $\underline{\text { StW352 }}>\mathrm{Gg}>\mathrm{Gb}>\mathrm{P}>\mathrm{B} \approx \mathrm{H}$ & $\mathrm{P}>\mathrm{B}>\mathrm{Gg}>\underline{\mathrm{StW}} 352>\mathrm{Gb}>$ \\
\hline
\end{tabular}

TCJ

$\mathrm{H}>\underline{\mathrm{StW} 352}>\mathrm{Gg}>\mathrm{P}>\mathrm{Gb} \quad \underline{\mathrm{StW} 352}>\mathrm{H}>\mathrm{Gg}>\mathrm{Gb} \approx \mathrm{P} \quad \mathrm{P}>\underline{\mathrm{StW} 352}>\mathrm{Gb}>\mathrm{Gg}>\mathrm{H}$

$\underline{\text { StW352 }}>\mathrm{Gg}>\mathrm{P}>\mathrm{Gb}>\mathrm{H}$

$\mathrm{P}>\mathrm{Gb}>\mathrm{H}>\mathrm{Gg}>\underline{\mathrm{StW} 352}$

${ }^{\mathrm{a}} \mathrm{DA}=$ degree of anisotropy, $\mathrm{E}=$ elongation index, $\mathrm{BV} / \mathrm{TV}=$ bone volume fraction, $\mathrm{Tb} . \mathrm{Th}=\mathrm{trabecular}$ thickness, Tb.N = trabecular number.

${ }^{\mathrm{b}} \mathrm{CCJ}=$ calcaneocuboid joint, TCJ = talocalcaneal joint.

${ }^{\mathrm{c}} \mathrm{H}=H$. sapiens, $\mathrm{P}=P$. troglodytes, $\mathrm{Gg}=G$. gorilla, $\mathrm{Gb}=G$. beringei, $\mathrm{B}=$ P. ursinus. 

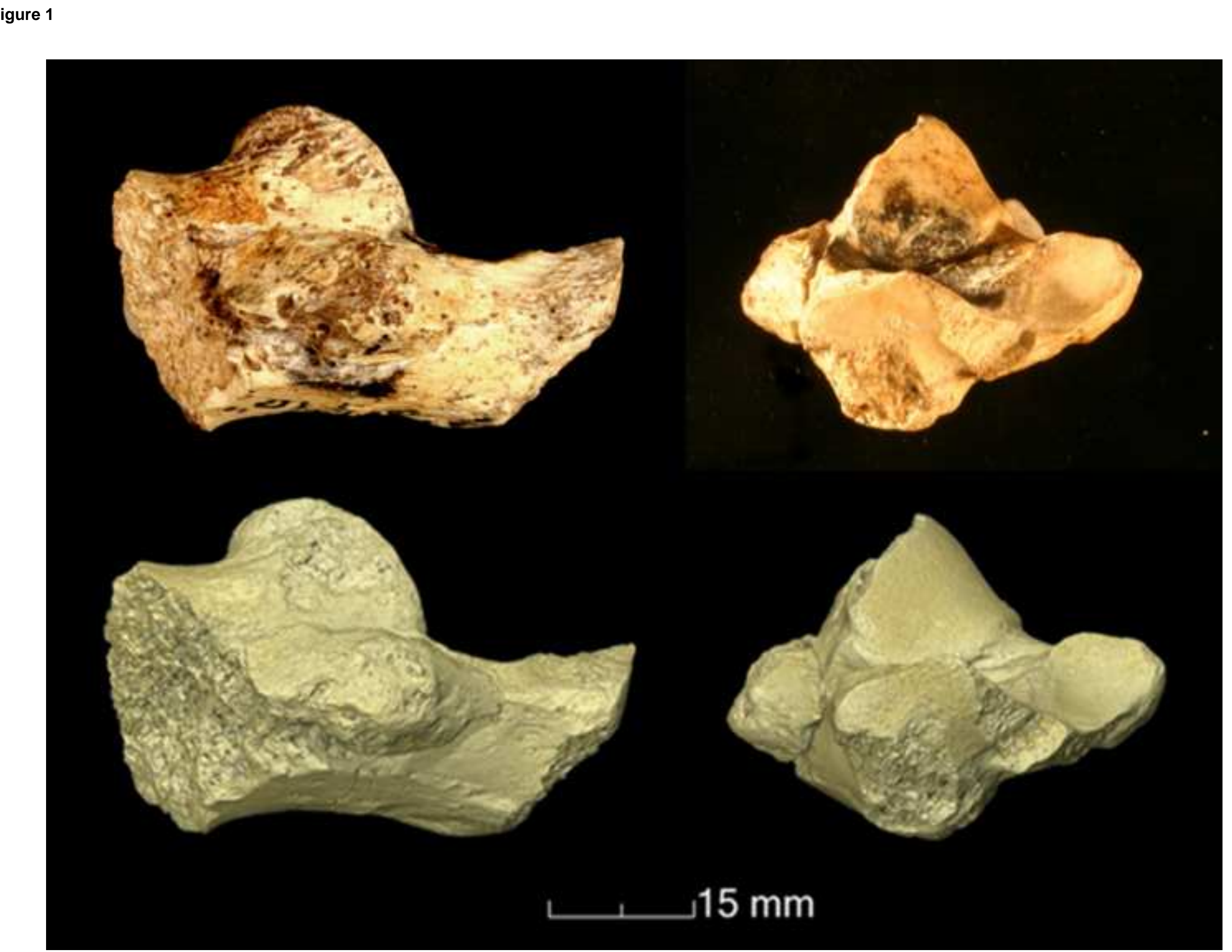

+ 15 mm
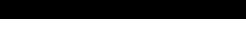

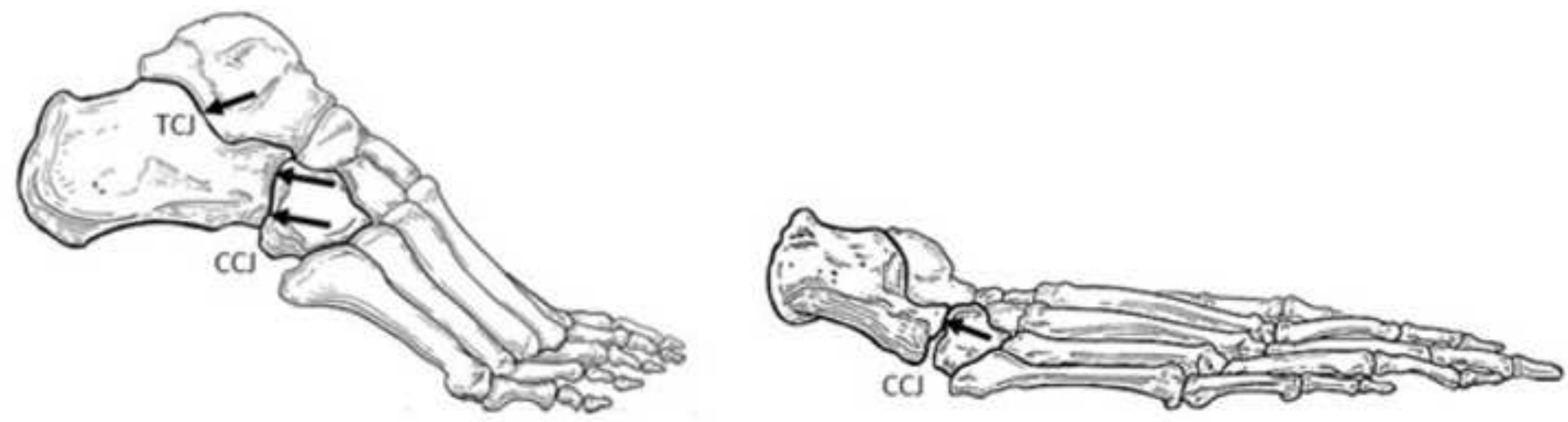


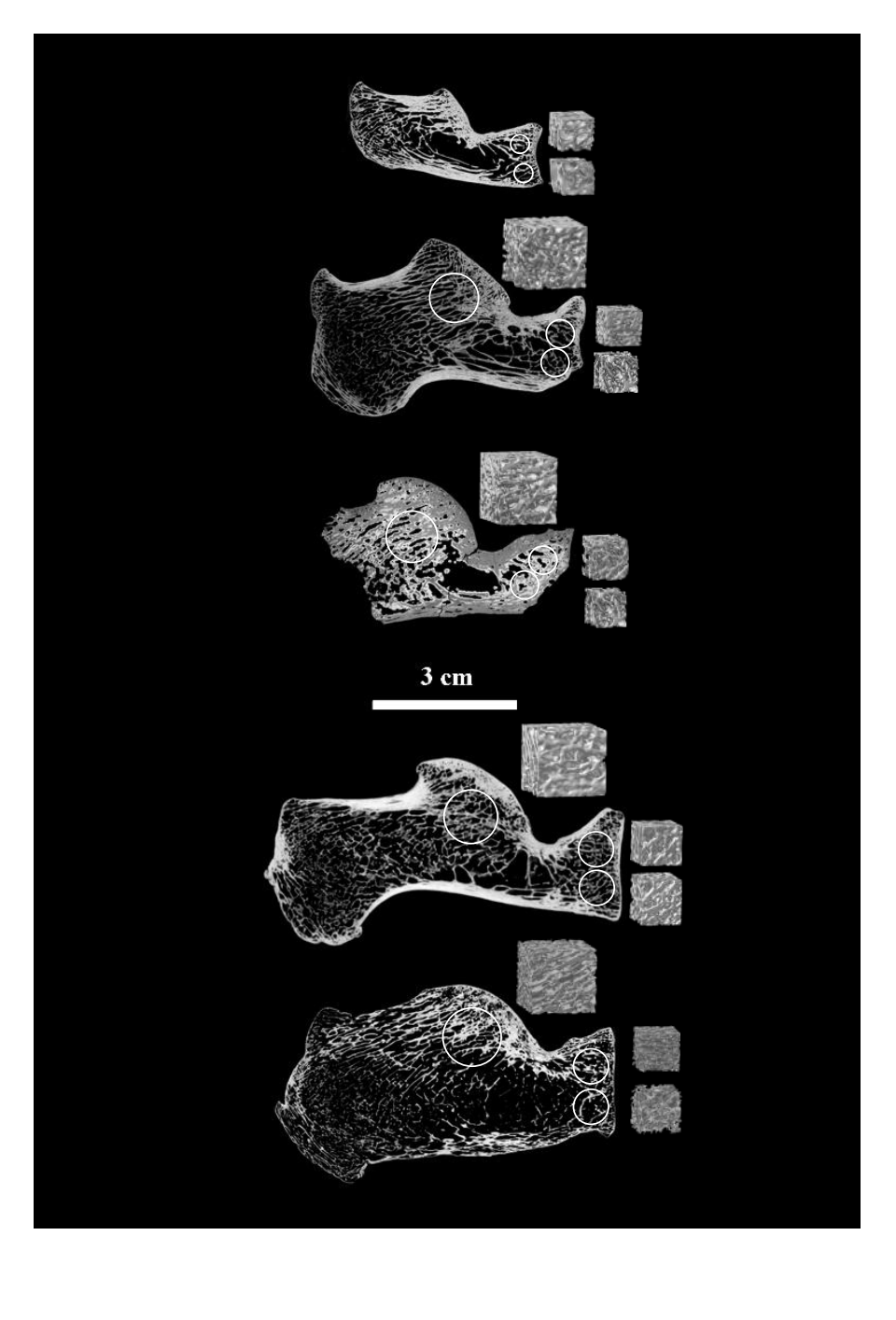

Figure 3

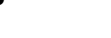

.

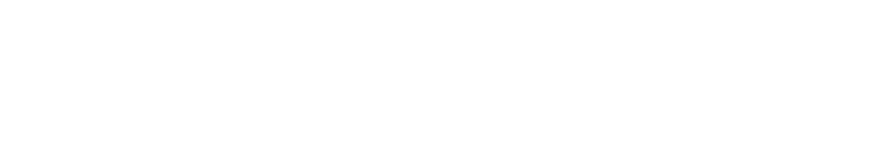
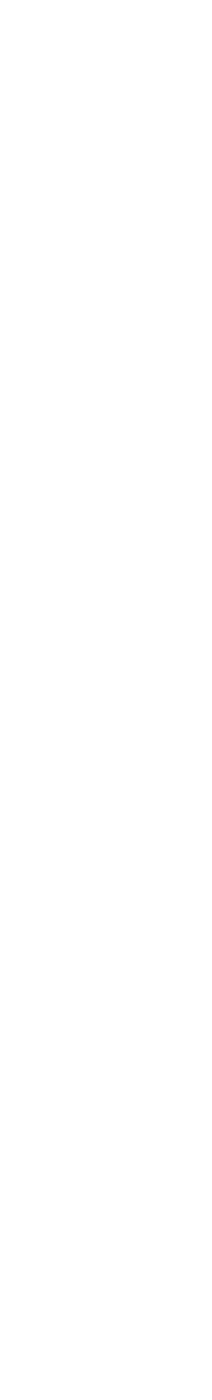


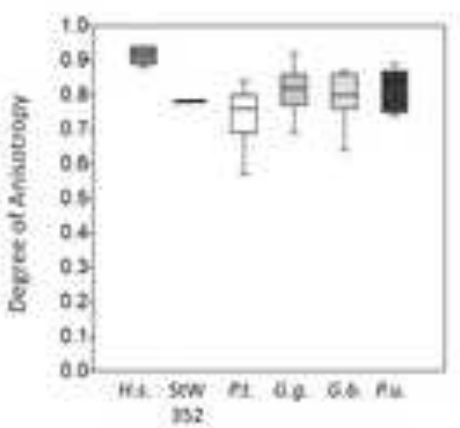

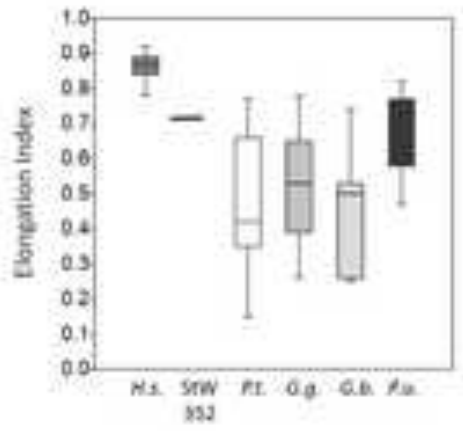

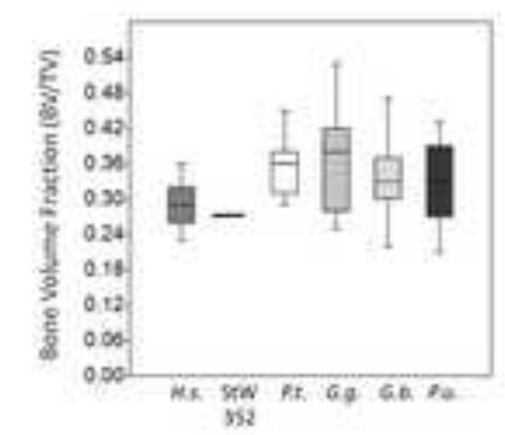

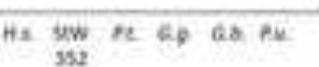

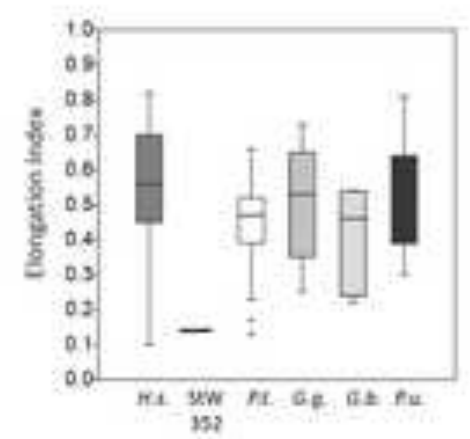

Plantar Calcanecocuboid Joint
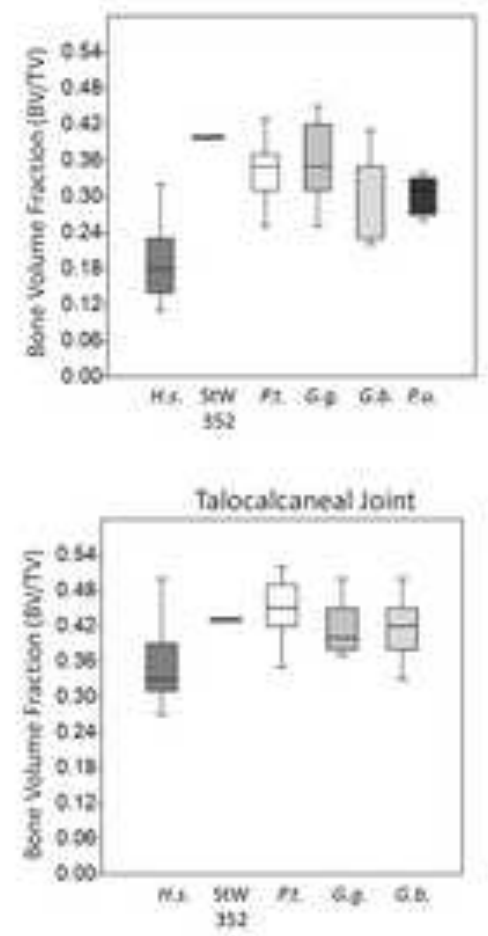
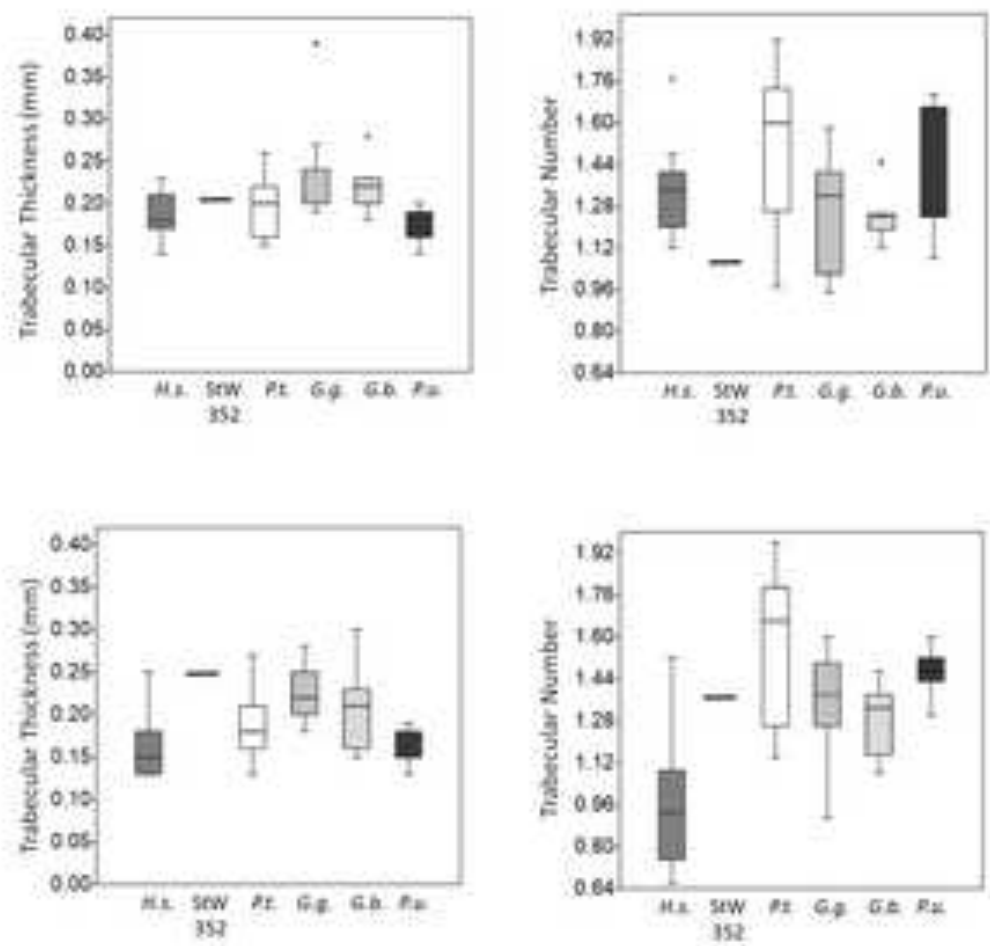

113

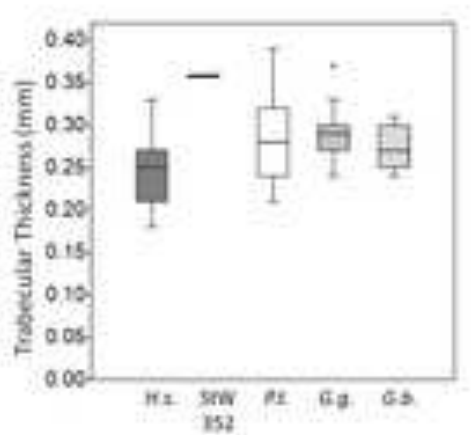

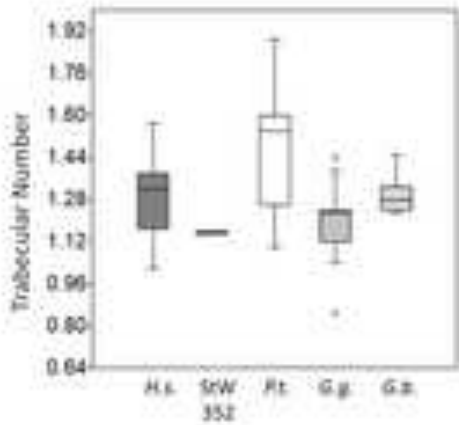


Figure 5

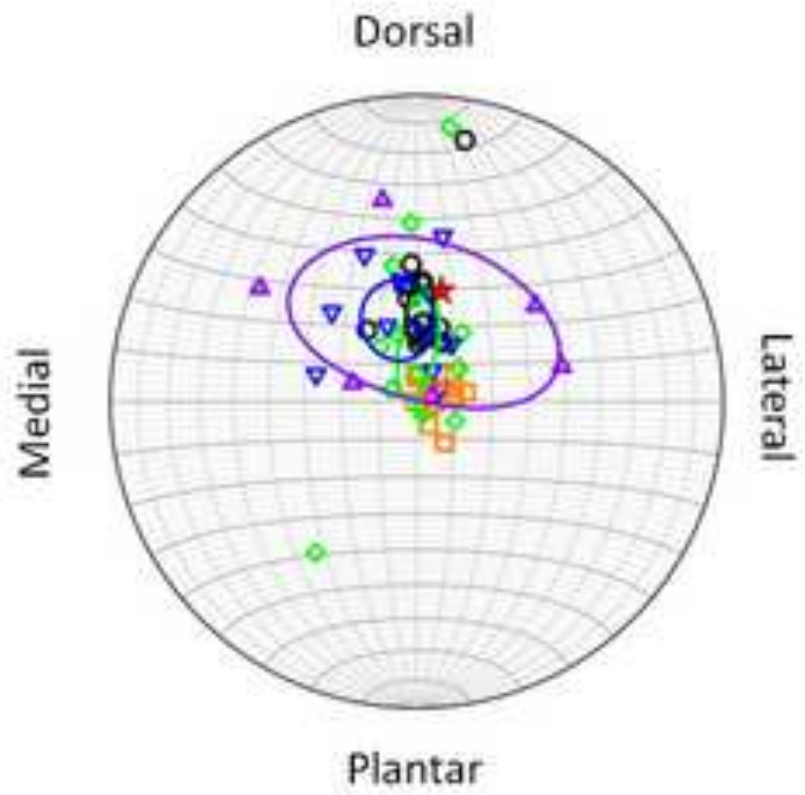
O H. sapiens
$\checkmark$ P. troglodytes

$\boldsymbol{\nabla}$ G. gorilla

$\Delta \mathrm{G}$, beringei

口 P. ursinus

$\star$ StW 352
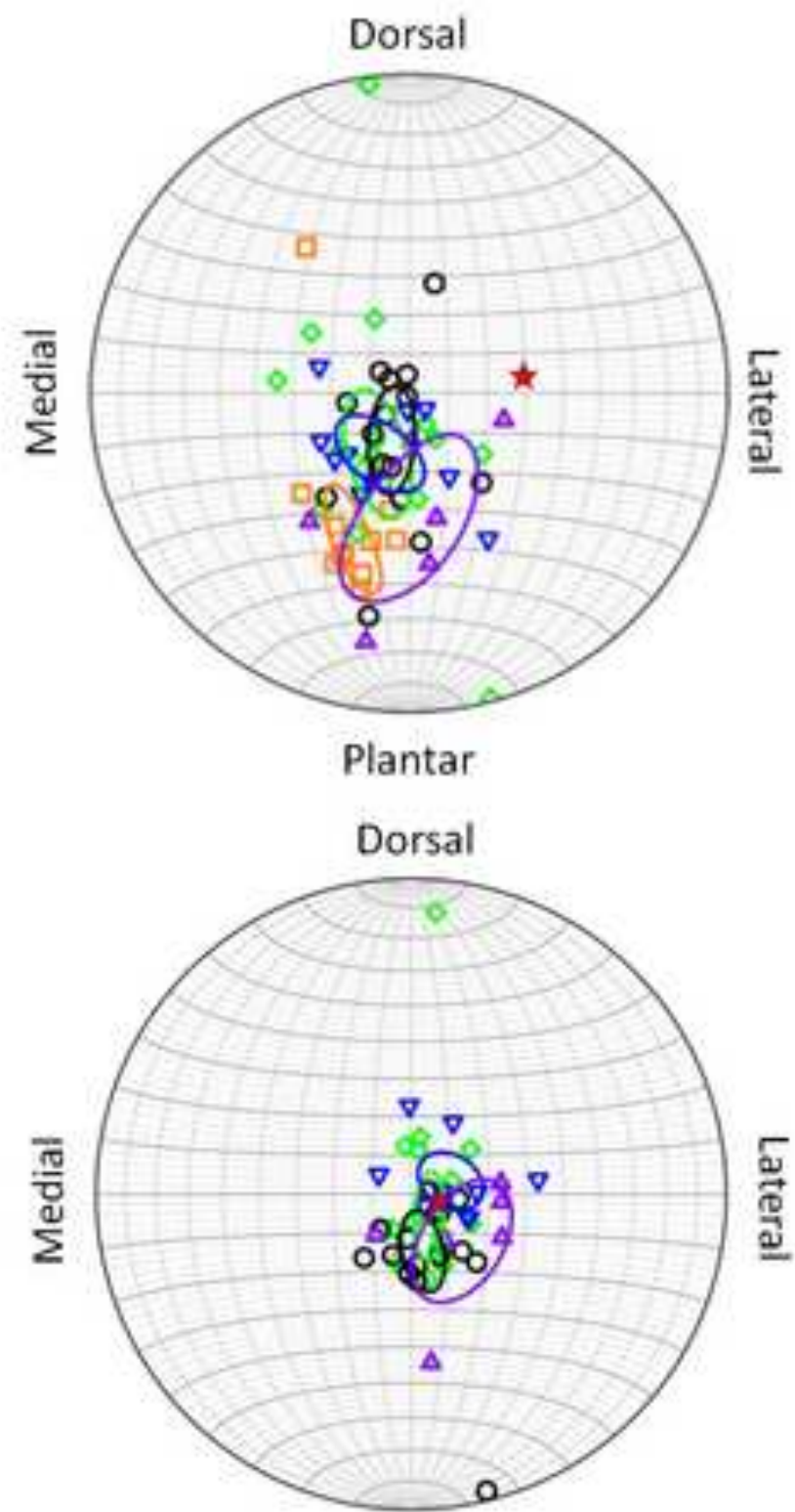

Plantar 